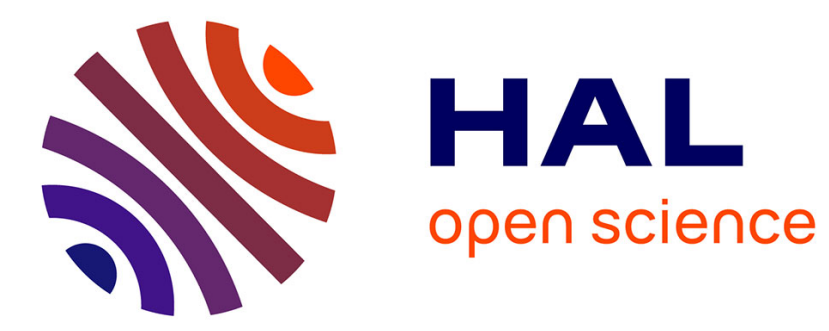

\title{
Maximizing points and coboundaries for an irrational rotation on the Circle
}

\author{
Julien Brémont
}

\section{To cite this version:}

Julien Brémont. Maximizing points and coboundaries for an irrational rotation on the Circle. ETDS, 2013, 33 (1), pp.24-48. hal-00794137

\section{HAL Id: hal-00794137 \\ https://hal.science/hal-00794137}

Submitted on 25 Feb 2013

HAL is a multi-disciplinary open access archive for the deposit and dissemination of scientific research documents, whether they are published or not. The documents may come from teaching and research institutions in France or abroad, or from public or private research centers.
L'archive ouverte pluridisciplinaire HAL, est destinée au dépôt et à la diffusion de documents scientifiques de niveau recherche, publiés ou non, émanant des établissements d'enseignement et de recherche français ou étrangers, des laboratoires publics ou privés. 


\title{
Maximizing points and coboundaries for an irrational rotation on the Circle
}

\author{
Julien Brémont†, and Zoltán Buczolichł† \\ † LAMA, Université Paris-Est \\ 61, avenue du Général de Gaulle, 94010 Créteil cedex, France \\ (e-mail: bremont@univ-paris12.fr) \\ $\ddagger$ Department of Analysis, Eötvös Loránd \\ University, Pázmány Péter Sétány 1/c, 1117 Budapest, Hungary \\ (e-mail:buczo@cs.elte.hu)
}

(Received September 5, 2011)

\begin{abstract}
Consider an irrational rotation of the unit circle and a real continuous function. A point is declared "maximizing" if the growth of the ergodic sums at this point is maximal up to an additive constant. In case of two-sided ergodic sums the existence of a maximizing point for a continuous function implies that it is the coboundary of a continuous function. In contrast, we build for the "usual" one-sided ergodic sums examples in Hölder or smooth classes indicating that all kinds of behaviour of the function with respect to the dynamical system are possible. We also show that generic continuous functions are without maximizing points, not only for rotations, but for the transformation $2 x \bmod 1$ as well. For this latter transformation it is known that any Hölder continuous function has a maximizing point.
\end{abstract}

\section{Introduction}

Let $(X, T)$ be a topological dynamical system, where $X$ is a compact metric space and $T: X \rightarrow X$ a continuous and surjective transformation.

Fix a continuous function $f: X \rightarrow \mathbb{R}$ and write $T f$ for $f \circ T$. Introduce the ergodic sums

$$
f_{n}(x)=\sum_{k=0}^{n-1} T^{k} f(x), \quad n \geq 1 .
$$

$\dagger$ Research supported by the Hungarian National Foundation for Scientific research K075242. 2000 Mathematics Subject Classification: Primary : 37A05, 37A25, 37E05; Secondary : 26A16. Keywords: maximizing point, coboundary, ergodic skew-product. 
The optimal pointwise growth of $\left(f_{n}(x)\right)$ is an important question arising naturally. We mention for example A. Cohen and J.-P. Conze [11] in a wavelet context, R. Mané [20] on Lagrangian systems, or optimization problems in computer science and algorithms when considering "discrete events systems", see D. A. Carlson, A. B. Haurie and A. Leizarowitz [8] or J. C. Lagarias and Y. Wang [17]. From these early works has emerged a field of research now called Ergodic Optimization, see T. Bousch [3], J.-P. Conze and Y. Guivarc'h [10] and the survey by O. Jenkinson [14]. The central question is the maximal mean growth of $\left(f_{n}(x)\right)_{n \geq 1}$, which is related to an optimization problem about invariant measures.

We focus in this article on a finer type of optimal growth and consider points $x \in X$ for which the growth of the sums $\left(f_{n}(x)\right)_{n \geq 1}$ is maximal up to an additive constant.

Definition 1. Let $f: X \rightarrow \mathbb{R}$ be continuous. A point $x_{0} \in X$ is "maximizing" for $f$ if there exists a constant $C \geq 0$ such that:

$$
\forall x \in X, \forall n \geq 1, \quad f_{n}(x) \leq f_{n}\left(x_{0}\right)+C .
$$

In this case, the shortfall of maximization at $x_{0}$ is

$$
S M\left(f, x_{0}\right)=\sup _{n \geq 1, x \in \mathbb{T}} f_{n}(x)-f_{n}\left(x_{0}\right) .
$$

We say that $x_{0}$ is "exactly maximizing" if one can take $C=0$ in (1) and "weakly maximizing" if $C$ is replaced by $C(x)$. If $\mu$ is a fixed Borel probability measure, we also say that $x_{0}$ is " $\mu$-weakly maximizing" if (1) is true for $\mu$-a.e $x$ with a constant $C(x)$.

The existence of maximizing points is naturally the first question to be adressed. If $f=c+g-T g$ with $g$ bounded and $c$ constant then clearly every point is maximizing for $f$. It is natural to ask whether this is the only situation.

The answer is negative for dynamical systems where the lemma of Mané-ConzeGuivarc'h is valid. In this case any Hölder continuous $f$ admits a maximizing point. Indeed, one can decompose $f=c+g-T g+r$, with $g$ and $r$ also Hölder continuous and verifying the properties that $r \leq 0$ and the level set $r^{-1}\{0\}$ carries a $T$-invariant set. A point in this set is maximizing for $f$ (in general the maximizing points for $f$ are not all contained in $r^{-1}\{0\}$ ). Systems where this lemma is true (see Bousch [4] for a recent result) satisfy some form of expansiveness and the classical thermodynamical formalism can be developed. A standard example is $T x=2 x \bmod (1)$ on $\mathbb{T}$.

For dynamical systems where such a decomposition result is not available, not much is known on the question of maximizing points. The purpose of the present article is to investigate this problem for an irrational rotation $T x=x+\alpha \bmod (1)$ on the unit circle $X=\mathbb{T}$.

We denote by $C_{m 0}(\mathbb{T})$ the set of those functions in $C(\mathbb{T})$ which have zero mean. In the same way we consider the spaces $C_{m 0}^{r}(\mathbb{T}), r \geq 1$, and the Hölder spaces $C_{m 0}^{\theta}(\mathbb{T}), 0<\theta<1$.

In this context, we mention first an interesting rigidity result for two-sided ergodic sums. The following lemma is taken from a manuscript of J-P. Conze, see also [9]. 
Lemma 2. Let $f \in C_{m 0}(\mathbb{T})$ and $T=T_{\alpha}$. If for some $x_{0} \in \mathbb{T}$

$$
\forall n \geq 1, \forall x \in \mathbb{T}, \quad \sum_{k=-n}^{n-1} T^{k} f(x) \leq \sum_{k=-n}^{n-1} T^{k} f\left(x_{0}\right)+C,
$$

then there exists $g \in C(\mathbb{T})$ such that $f=g-T g$.

Proof. Taking $x=T^{k} x_{0}$ with $k \geq 0$, we get by cancellation of terms $f_{k}\left(T^{n} x_{0}\right) \leq$ $f_{k}\left(T^{-n} x_{0}\right)+C$. Using density of $\left(T^{n} x_{0}\right)$ and continuity of $f$, for all $k \geq 0$ and $y \in \mathbb{T}$, $f_{k}\left(x_{0}+y\right) \leq f_{k}\left(x_{0}-y\right)+C$. By symmetry

$$
\forall k \geq 0, \forall y \in \mathbb{T}, \quad\left|f_{k}\left(x_{0}+y\right)-f_{k}\left(x_{0}-y\right)\right| \leq C
$$

Using the cocycle property of $\left(f_{n}\right)$ (see $\left.(3)\right)$ and again the continuity, $\mid f_{n}(x+y)-f_{n}(x-$ $y) \mid \leq 2 C$, for all $n \geq 0$ and $x, y \in \mathbb{T}$. This can be rewritten as

$$
\forall n \geq 0, \forall x, y \in \mathbb{T}, \quad\left|f_{n}(x)-f_{n}(y)\right| \leq 2 C .
$$

Since $f_{n}$ has zero mean, $f_{n}\left(y_{n}\right)=0$ for some $y_{n}$ and hence $\left|f_{n}(x)\right| \leq 2 C$. As a result $f=g-T g$ with continuous $g$ by Gottschalk and Hedlund's Theorem (cf [13], Theorem 14.11).

One deduces that continuous functions with a maximizing point $x_{0}$ and presenting a symmetry with respect to $x_{0}$ show similar behaviour.

COROllary 3. Let $f \in C_{m 0}(\mathbb{T})$ have a maximizing point $x_{0}$. If $f\left(x_{0}+x\right)=f\left(x_{0}-x\right)$ for all $x \in \mathbb{T}$, then $f=g-T g$ for some $g \in C(\mathbb{T})$.

Proof. For $x \in \mathbb{T}$ and $n \geq 1$

$$
\begin{aligned}
\sum_{k=-n}^{n-1} T^{k} f(x) & =f_{n}(x)+f_{n}\left(2 x_{0}-x\right)+T^{n} f\left(2 x_{0}-x\right)-f\left(2 x_{0}-x\right) \\
& \leq 2 f_{n}\left(x_{0}\right)+2 C+2\|f\|_{\infty} .
\end{aligned}
$$

Since $\sum_{k=-n}^{n-1} T^{k} f\left(x_{0}\right)=2 f_{n}\left(x_{0}\right)+T^{n} f\left(x_{0}\right)-f\left(x_{0}\right) \geq 2 f_{n}\left(x_{0}\right)-2\|f\|_{\infty}$, the result follows from Lemma 2.

The assumption of symmetry is of course very strong. We will show that the situation can be very different. Hölder or smooth functions may not have a maximizing point and the ones with a maximizing point are not reduced to those of the form $f=c+g-T g$, with $g$ continuous and $c$ constant. In fact we prove something stronger in the classes $\cap_{0<\theta<1} C_{m 0}^{\theta}(\mathbb{T})$ and $C_{m 0}^{r}(\mathbb{T}), r \geq 1$, supposing in this latter case that $\alpha$ has Diophantine type larger than $r$. The following mutually excluding cases are all possible:

- There exists $f$ with zero mean, having a maximizing point and such that $f$ defines an ergodic skew-product. In particular, no measurable $g$ verifies $f=g-T g$, a.e.

- There exists $f=g-T g$ with a maximizing point, where moreover $g \in$ $\cap_{1<p<\infty} L^{p}(\mathbb{T}) \backslash L^{\infty}(\mathbb{T})$ (and thus $g$ is not continuous). 
- There are functions without any maximizing point.

This opens perspectives of research, because the condition of having a maximizing point seems compatible with any behaviour of the function with respect to the dynamical system. It would be interesting in a future work to give ways of characterizing maximizing points or sufficient conditions for their existence.

We next consider generic results. In the class $C(\mathbb{T})$, we show that a generic function has no weakly maximizing point. Adapting our method, we prove a similar result for the dynamical system $T x=2 x \bmod (1)$ on $\mathbb{T}$.

We conclude the article with some complementary results on the set of maximizing points and finally list several open questions.

\section{2. $\quad$ Notation and conventions}

- Let $\mathbb{N}=\{1,2, \ldots$.$\} . The unit circle \mathbb{T}$ is identified with $\mathbb{R} / \mathbb{Z}$. Lebesgue measure on $\mathbb{T}$ is denoted by $\lambda_{\mathbb{T}}$ (or simply $\lambda$ ) and on $\mathbb{R}$ by $\lambda_{\mathbb{R}}$.

- For $f: \mathbb{T} \rightarrow \mathbb{R}$ we introduce the cocycle notation

$$
f_{n}(x)=\left\{\begin{array}{cc}
\sum_{k=0}^{n-1} T^{k} f(x), & n \geq 1 \\
0, & n=0 \\
-\sum_{k=n}^{-1} T^{k} f(x), & n \leq-1
\end{array}\right.
$$

We have

$$
f_{n+m}(x)=f_{n}(x)+f_{m}\left(T^{n} x\right) \text { for any } n, m \in \mathbb{Z} \text {. }
$$

- We say that $f$ is a coboundary if $f=g-T g$ for some measurable $g$ and that $f$ is a $\mathcal{C}$-coboundary if moreover $g \in \mathcal{C}$, for some class $\mathcal{C}$.

- By our convention any sum $\sum_{m}^{n}$ with $n<m$ is equal to 0 .

- For $x \in \mathbb{R}$, let $\|x\|$ denote the distance from $x$ to the nearest integer.

- Suppose we have an irrational number $\alpha \in[0,1)$, then its continued fraction development is

$$
\alpha=\left[a_{1}, a_{2}, \ldots\right]=\frac{1}{a_{1}+\frac{1}{a_{2}+\frac{1}{\ldots}}}, \text { with } a_{n} \in \mathbb{N} .
$$

The convergents of $\alpha$ are given by the rational numbers $\left(p_{n} / q_{n}\right)$ with the terminating continued fraction development $p_{n} / q_{n}=\left[a_{1}, a_{2}, \ldots, a_{n}\right]$. We will use that $1 /\left(2 q_{n+1}\right) \leq\left\|q_{n} \alpha\right\| \leq 1 / q_{n+1},\|k \alpha\| \geq\left\|q_{n} \alpha\right\|$, for $1 \leq k<q_{n+1}$ and $q_{n} \geq 2^{(n-1) / 2}$ (see for example $[\mathbf{1 5}])$. 
- The Diophantine type of $\alpha$ is

$$
\eta(\alpha)=\sup \left\{s>0, \liminf q^{s}\|q \alpha\|=0\right\} .
$$

Recall that $\eta(\alpha) \geq 1$ for any irrational $\alpha$. It is a standard result that almost-every $\alpha$ has a Diophantine type equal to 1 . This is the case for example for rotation angles with bounded partial quotients.

\section{Main results}

Recall that if $f=g-T g$ for a measurable $g$ then by ergodicity $g$ is unique up to an additive constant and a null set.

For Hölder continuous functions, we will prove the following result :

Theorem 4. Let $T x=x+\alpha \bmod (1)$ on $\mathbb{T}, \alpha \notin \mathbb{Q}$. Any of the following mutually excluding conditions is realized by at least one $f \in \cap_{0<\theta<1} C_{m 0}^{\theta}(\mathbb{T})$.

i) The point 0 is exactly maximizing for $f$, that is,

$$
\forall x \in \mathbb{T}, \forall n \geq 0, \quad f_{n}(x) \leq f_{n}(0)
$$

and there exists $g \in \cap_{1<p<\infty} L^{p}(\mathbb{T}) \backslash L^{\infty}(\mathbb{T})$ such that $f=g-T g$, a.e. In particular, $g$ is not continuous.

ii) The point 0 is exactly maximizing for $f$

$$
\forall x \in \mathbb{T}, \forall n \geq 0, \quad f_{n}(x) \leq f_{n}(0)
$$

and the skew-product $\left(\mathbb{T} \times \mathbb{R}, T_{f}, \lambda_{\mathbb{T}} \otimes \lambda_{\mathbb{R}}\right)$ defined by $f$ is ergodic, where $T_{f}(x, y)=$ $(T x, y+f(x))$. In particular $f$ is not a measurable coboundary.

iii) Let $\varepsilon(n) \searrow 0$ as $n \nearrow+\infty$. For any $x \in \mathbb{T}$ for a.e $y \in \mathbb{T}$

$$
\sup _{n \in \mathbb{N}}\left\{n^{-\varepsilon(n)}\left(f_{n}(y)-f_{n}(x)\right)\right\}=+\infty .
$$

In particular $f$ does not have any $\lambda$-weakly maximizing point.

REMARK 5. The first case of the theorem is in some sense the first non-trivial situation for a coboundary (the trivial one being here when $g \in L^{\infty}(\mathbb{T})$, but then the identically zero function is a good example verifying (4)). In the second case the behaviour of the ergodic sums is much wilder. By Theorem 14.13 of [13] one may notice that the function $f$ in i) defines a topologically transitive skew product. 
REMARK 6. In Theorem 4 ii), $f$ cannot be absolutely continuous with $f^{\prime} \in L^{2}$ (for example $f$ Lipschitz) without imposing Diophantine conditions on $\alpha$, since if $f^{\prime} \in L^{2}$ it is classical that $f=g-T g$ with $g \in L^{2}$ when $\alpha$ has bounded partial quotients. Indeed, defining $g$ by its Fourier coefficients $(\hat{g}(n))$, we have $\hat{g}(n)=\hat{f}(n) /\left(1-e^{2 i \pi n \alpha}\right)$ for $n \neq 0$. Thus $|\hat{g}(n)| \leq C\left|\hat{f}^{\prime}(n)\right| /(|n|\|n \alpha\|)$. The claim follows from $\left(\hat{f}^{\prime}(n)\right) \in \ell^{2}$ and $|n|\|n \alpha\| \geq c>0$.

Let us mention in passing a strong obstruction for $C^{1}$-regularity.

LEMMA 7. A function $f \in C^{1}(\mathbb{T})$ has no exactly maximizing point unless it is constant.

Proof. We prove a slightly stronger version : if there are $x_{0} \in \mathbb{T}$ and $\delta_{0}>0$ such that $f_{n}(x) \leq f_{n}\left(x_{0}\right)$, for every $x \in\left(x_{0}-\delta_{0}, x_{0}+\delta_{0}\right)$ and $n \geq 1$, then $f$ is constant.

Proceeding towards a contradiction, suppose that there is an $n \geq 1$ such that $f^{\prime}\left(T^{n} x_{0}\right) \neq 0$ and take the first $n$ with this property. Since the other case is very similar, without limiting generality, we suppose that $f^{\prime}\left(T^{n} x_{0}\right)>0$. We can then choose $\delta \in\left(0, \delta_{0}\right)$ such that for $x_{0}<x<x_{0}+\delta$

$$
f_{n+1}(x)>f_{n+1}\left(x_{0}\right)+\left(x-x_{0}\right) f^{\prime}\left(T^{n} x_{0}\right) / 2>f_{n+1}\left(x_{0}\right)
$$

contradicting $f_{n+1}(x) \leq f_{n+1}\left(x_{0}\right)$. As a result, $f^{\prime}\left(T^{n} x_{0}\right)=0$ for all $n \geq 1$. As $\left(T^{n} x_{0}\right)_{n \geq 0}$ is dense in $\mathbb{T}$ and $f^{\prime}$ is continuous, $f^{\prime}$ is identically 0 .

REMARK 8. When $f \in \cap_{0<\theta<1} C^{\theta}(\mathbb{T})$ its Fourier coefficients satisfy $\hat{f}(n)=O\left(|n|^{-\theta}\right)$, for every $0<\theta<1$. Proceeding for example as in corollary 3.2 of J. Aaronson, M. Lemańczyk, C. Mauduit and H. Nakada [1], one gets $\left\|f_{q_{n}}\right\|_{L^{2}(\mathbb{T})}=O\left(q_{n}^{\varepsilon}\right)$, for any $\varepsilon>0$. If $\alpha$ has Diophantine type 1 , one obtains (cf. for instance $[\mathbf{9}]$, section 2.1) $\left\|f_{n}\right\|_{L^{2}(\mathbb{T})}=O\left(n^{\varepsilon}\right)$, for every $\varepsilon>0$. One can compare it to the rate in Theorem 4 iii).

REMARK 9. About iii), in the present context of an irrational rotation on $\mathbb{T}$, the notion of weakly maximizing point reduces to that of maximizing point. Indeed, if some $x_{0}$ verifies

$$
\forall y \in \mathbb{T}, S M\left(f, x_{0}, y\right)<+\infty \text {, with } S M\left(f, x_{0}, y\right)=\sup _{n \geq 1} f_{n}(y)-f_{n}\left(x_{0}\right),
$$

then for all $M \geq 0, A_{M}:=\left\{y \in \mathbb{T} \mid S M\left(f, x_{0}, y\right) \leq M\right\}$ is closed and $\mathbb{T}=\cup_{M \in \mathbb{N}} A_{M}$. By Baire's theorem, some $A_{M_{0}}$ has non-empty interior. Consequently, the entrance time $\tau(y)$ of $y$ in $A_{M_{0}}$ is uniformly bounded in $y$, by some constant $T_{0}$. Then clearly

$$
\forall y \in \mathbb{T}, S M\left(f, x_{0}, y\right) \leq M_{0}+2\|f\|_{\infty} T_{0}
$$

We next consider smooth examples. We write $\left(a_{n}\right)$ for the partial quotients of $\alpha$ and $\left(p_{n} / q_{n}\right)$ for its sequence of convergents. We prove an analogous theorem for smooth functions.

ThEOREM 10. If $r \in \mathbb{N}$ and $\sup _{m \geq 1} a_{m+1} q_{m}^{-r+1}=+\infty$, then any of the following mutually excluding conditions is satisfied by at least one $f \in C_{m 0}^{r}(\mathbb{T})$. 
i) The point 0 is a maximizing point for $f$ and $f$ has the form $f=g-T g$, with $g \in \cap_{1<p<\infty} L^{p}(\mathbb{T}) \backslash L^{\infty}(\mathbb{T})$.

ii) The point 0 is a maximizing point for $f$ and the skew-product $\left(\mathbb{T} \times \mathbb{R}, T_{f}, \lambda_{\mathbb{T}} \otimes \lambda_{\mathbb{R}}\right)$ defined by $f$ is ergodic.

iii) The function $f$ has no maximizing point.

If $\sup _{m \geq 1} \log a_{m+1} / \log q_{m}=+\infty$, then $f$ can be chosen in $C_{m 0}^{\infty}(\mathbb{T})$.

REMARK 11. In the $C^{r}$-part of the theorem the Diophantine condition is verified as soon as $\eta(\alpha)>r$. This is close to optimal, since when $f \in C_{m 0}^{r}(\mathbb{T})$ and $\eta(\alpha)<r$, then $f=g-T g$, for some $g \in C(\mathbb{T})$, cf V. Arnold [2].

REMARK 12. We discuss the case of real-analytic examples in the final section.

In section 6 generic functions in $C(\mathbb{T})$ are considered. We prove the following theorem: TheOREM 13. Let $T x=x+\alpha \bmod (1)$ on $\mathbb{T}$, with $\alpha \notin \mathbb{Q}$. Then a generic function in $C(\mathbb{T})$ has no weakly maximizing point.

Our techniques also allow to treat the case of a dynamical system with a very different nature. We show:

Theorem 14. Let $T x=2 x \bmod (1)$ on $\mathbb{T}$. Then a generic function in $C(\mathbb{T})$ has no maximizing point.

4. Hölder continuous examples

We first consider the proof of Theorem 4. The following key lemma furnishes Lipschitz coboundaries $f=g-T g$ with $\|f\|_{\infty}$ small compared to $\|g\|_{\infty}$ and such that 0 is an exactly maximizing point for $f$.

Lemma 15. Suppose $B>0$ and $N \in \mathbb{N}$ are given. Let $I=[-\Delta, \Delta]$, where $\Delta>0$ is such that the $2 I+k \alpha,-N \leq k<N$, are disjoint on $\mathbb{T}$. Define

$$
F=\bigcup_{-N \leq k<N} I+k \alpha
$$

Introduce the peak function $\bar{f}(x)=B \cdot(1-|x| / \Delta)_{+}$and set

$$
f=\sum_{k=0}^{N-1} T^{-k} \bar{f}-\sum_{k=1}^{N} T^{k} \bar{f} .
$$

Notice that $f=h-T h$, where $h$ is Lipschitz continuous and given by

$$
h=N \bar{f}+\sum_{l=1}^{N-1}(N-l)\left(T^{-l} \bar{f}+T^{l} \bar{f}\right) .
$$

Then $f$ has the following properties :

Prepared using etds.cls 


$$
\begin{gathered}
|f(x)| \leq B, 0 \leq h(x) \leq N B, \forall x \in \mathbb{T}, \\
\text { if } n \in \mathbb{N} \text { and } x \in \mathbb{T},\left|f_{n}(x)\right| \leq N B, \\
|f(x)-f(y)| \leq(B / \Delta)|x-y|, \forall x, y \in \mathbb{T}, \\
\text { if } 0 \leq n \leq N, \text { then } f_{n}(0)=n B, \\
\text { if } n \in \mathbb{N}, x \notin F, \text { and } T^{n} x \notin F, \text { then } f_{n}(x)=0, \\
\text { if } n \in \mathbb{N} \text { and } x \notin F \text { then }-N B \leq f_{n}(x) \leq 0 \leq f_{n}(0), \\
\text { if } n \in \mathbb{N} \text { and } x \in \mathbb{T} \text { then } f_{n}(x) \leq f_{n}(0) .
\end{gathered}
$$

Proof. Since $f_{n}(x)=h(x)-T^{n} h(x), 0 \leq h(x) \leq N B=h(0)$ and $h(y)=0$ when $y \notin F$, the properties except (14) follow directly from the definition of $\bar{f}$ and $f$.

The last one, (14), is more delicate. It is a consequence of

$$
\forall x, y \in \mathbb{T}, \quad h(0)-h(x) \geq h(y)-h(x+y)
$$

To show this, fix $x$ and take $y$ realizing the maximum of $h(y)-h(y+x)$. As $y \longmapsto$ $h(y)-h(y+x)$ is piecewise linear, we assume that $y$ or $y+x$ is an angular point for $h$. Suppose also that $x, y$ are interior to $\cup_{|m|<N} I+m \alpha$, otherwise (15) is evident. Let then $x \in I+k \alpha$ and $y \in I+l \alpha$, with $|k|,|l|<N$. Since $h(0)=N B$ and $h(x) \leq B(N-|k|)$, $h(y) \leq B(N-|l|)$, we suppose that $|k|+|l|<N$. We distinguish two cases.

1) As $h(y)>0$, if $y$ is an angular point, then $y=l \alpha$. In this case, $h(0)-h(y)=|l| B$, whereas $h(x)-h(x+y)=f_{l}(x) \leq|l| B$, so we have (15).

2) Suppose now that $y+x$ is an angular point for $h$, but $y$ is not. If $y+x$ corresponds to a peak, one can move $y$ a little so that $h(y)-h(x+y)$ is strictly larger. Therefore $y+x$ is at the basis of a peak, whose top is at $(k+l) \alpha$, since the $2 I+m \alpha,|m|<N$, are disjoint. We have $x=k \alpha+r, y=l \alpha+s$, with $|r|<\Delta,|s|<\Delta$ and $r+s= \pm \Delta$ in order to satisfy the condition on $x+y$. In particular, $|r|+|s|=\Delta$. We get

$$
\begin{aligned}
h(x)+h(y) & =B(N-|k|)\left(1-\frac{|r|}{\Delta}\right)+B(N-|l|)\left(1-\frac{|s|}{\Delta}\right) \\
& \leq N B-B|k|\left(1-\frac{|r|}{\Delta}\right)-B|l|\left(1-\frac{|s|}{\Delta}\right) \leq h(0) .
\end{aligned}
$$

This completes the proof of the lemma.

Proof of Theorem 4. We use the denominators $\left(q_{n}\right)_{n \geq 1}$ of the convergents of $\alpha$. Recall that for all $0<k<q_{n}$, we have $\|k \alpha\|>1 /\left(2 q_{n}\right)$.

Case i). For $m \geq 1$, set $N_{m}=\left\lfloor q_{m} / 4\right\rfloor$ and $B_{m}=m / q_{m}$. Let $f^{1}=f^{2}=f^{3}=f^{4}=0$. When $m \geq 5$, we have $N_{m} \geq 1$ and in Lemma 15 with $\left(B_{m}, N_{m}\right)$, we choose $\Delta_{m}=$ $e^{-\sqrt{m}} /\left(8 q_{m}\right)$. We get $\bar{f}^{m}$ and next $f^{m}$ with support $F_{m}$. 
Put $f=\sum_{m=1}^{\infty} f^{m}$. Since $\left|f^{m}\right| \leq B_{m}$, via (8) and $\sum B_{m}<\infty, f$ is continuous. By (7) and (10), $f^{m}$ has the Lipschitz constant $B_{m} / \Delta_{m}=8 m e^{\sqrt{m}}$. Fix $0 \leq \theta<1$. Since $q_{m}$ grows at least exponentially fast, for $m$ large enough

$$
\frac{B_{m}}{\Delta_{m}}\left(\tau \Delta_{m}\right)=8 m e^{\sqrt{m}}\left(\tau \Delta_{m}\right) \leq 8 m e^{\sqrt{m}}\left(\Delta_{m}\right)^{1-\theta}\left(\tau \Delta_{m}\right)^{\theta} \leq \frac{1}{m^{2}}\left(\tau \Delta_{m}\right)^{\theta}
$$

for all $0 \leq \tau \leq 1$.

Hence,

$$
\left|f^{m}(x)-f^{m}(y)\right| \leq \frac{1}{m^{2}}|x-y|^{\theta} \text { for }|x-y|=\tau \Delta_{m} \leq \Delta_{m}
$$

On the other hand, if $|x-y|>\Delta_{m}$ then for sufficiently large $m$

$$
\left|f^{m}(x)-f^{m}(y)\right| \leq 2 B_{m} \leq \frac{1}{m^{2}} \Delta_{m}^{\theta} \leq \frac{1}{m^{2}}|x-y|^{\theta} .
$$

Therefore, for large $m,\left|f^{m}(x)-f^{m}(y)\right| \leq\left(1 / m^{2}\right)|x-y|^{\theta}$, for every $x, y \in \mathbb{T}$. Adding these inequalities we obtain $f \in C^{\theta}(\mathbb{T})$. Finally, we have $f \in \cap_{0<\theta<1} C^{\theta}(\mathbb{T})$.

Condition (4) is true for every $f^{m}$, by (14), and so is verified by $f$.

We next show that $f=g-T g$, a.e., with $g \in \cap_{1<p<\infty} L^{p}(\mathbb{T})$. Via for example [7] or [19], it is enough to establish that for all $1<p<\infty$

$$
\sup _{n \geq 0}\left\|f_{n}\right\|_{p}<\infty
$$

Recall that by (6) and (7), $\lambda\left(F_{m}\right)=2 \Delta_{m} N_{m}$. Since $\sum_{m \geq 1} \Delta_{m} N_{m}<\infty$, we define for $N \geq 1$

$$
A_{N}=\cap_{m>N}\left(F_{m}\right)^{c} \text { and } R_{N}=\sum_{m>N} \Delta_{m} N_{m}<\sum_{m>N} e^{-\sqrt{m}} .
$$

Note that $\sum_{1 \leq m \leq N} N_{m} B_{m} \leq \sum_{1 \leq m \leq N} m / 4 \leq N^{2}$. Setting $\varepsilon=4 R_{N}$, we have $\lambda\left(A_{N}\right) \geq 1-\varepsilon / 2$. If $n \geq 0$, we obtain $\lambda\left(A_{N} \cap T^{-n} A_{N}\right) \geq 1-\varepsilon$.

Suppose $x \in A_{N} \cap T^{-n} A_{N}$. Since $x \notin F_{m}$ and $T^{n} x \notin F_{m}$ for $m>N$ we have for these $m$ 's, $f_{n}^{m}(x)=0$, by (12). Therefore, for such an $x$

$$
f_{n}(x)=\sum_{m \geq 1} f_{n}^{m}(x)=\sum_{m=1}^{N} f_{n}^{m}(x)
$$

Hence for all $n \geq 0, \lambda\left\{x \in \mathbb{T}:\left|f_{n}(x)\right| \leq \sum_{1 \leq m \leq N} N_{m} B_{m}\right\} \geq 1-\varepsilon$ and thus, $\lambda\left\{x \in \mathbb{T}:\left|f_{n}(x)\right| \geq N^{2}\right\} \leq 4 R_{N}$. Using this inequality, we can write:

$$
\begin{aligned}
\int\left|f_{n}\right|^{p} d \lambda & =p \int_{0}^{+\infty} \lambda\left\{\left|f_{n}\right|>t\right\} t^{p-1} d t \\
& \leq p\left(1+\sum_{N \geq 1} \int_{N^{2}}^{(N+1)^{2}} \lambda\left\{\left|f_{n}\right|>t\right\} t^{p-1} d t\right) \\
& \leq p\left(1+4 \sum_{N \geq 1} R_{N}(1+2 N)(N+1)^{2(p-1)}\right)<\infty
\end{aligned}
$$

Prepared using etds.cls 
due to (20). This gives (19) and thus $f=g-T g$, a.e, with $g \in \cap_{1<p<\infty} L^{p}(\mathbb{T})$.

Next we prove that $g \notin L^{\infty}(\mathbb{T})$. Proceeding towards a contradiction, suppose that $g \in L^{\infty}(\mathbb{T})$. Then $g$ is uniformly bounded in a $T$ invariant set of full measure. Therefore, for some $x$ the sequence $f_{n}(x)$ is uniformly bounded for all integers $n$. The theorem of Gottschalk and Hedlund (Theorem 14.11 of $[\mathbf{1 3}]$ ) implies that $g=\tilde{g}$, a.e, where $\tilde{g} \in C(\mathbb{T})$. Thus $f=\tilde{g}-T \tilde{g}$ everywhere on $\mathbb{T}$ and $\left\|f_{n}\right\|_{\infty} \leq 2\|\tilde{g}\|$, for all $n \geq 0$. We establish a contradiction by showing

$$
\sup _{n \in \mathbb{N}}\left\|f_{n}\right\|_{\infty}=\infty
$$

Using (13), for all $m \geq 1, n \geq 0$, we have $\left(f^{m}\right)_{n}(0) \geq 0$. Also by $(11),\left(f^{n}\right)_{N_{n}}(0)=$ $N_{n} B_{n} \geq n / 8$ for large $n$. Then

$$
f_{N_{n}}(0)=\sum_{m \geq 1}\left(f^{m}\right)_{N_{n}}(0) \geq\left(f^{n}\right)_{N_{n}}(0) \geq n / 8 .
$$

As $n$ is arbitrary large, this proves (21) and completes the proof of Case i).

Case ii). We first detail a classical strategy for proving the ergodicity with respect to Lebesgue measure $\lambda_{\mathbb{T}} \otimes \lambda_{\mathbb{R}}$ on $\mathbb{T} \times \mathbb{R}$ of a skew-product $T_{f}: \mathbb{T} \times \mathbb{R} \rightarrow \mathbb{T} \times \mathbb{R}$ associated to a real measurable $f$.

For such a dynamical system (where the invariant measure is infinite) the problem of ergodicity can be reformulated using the concept of essential value, see K. Schmidt [22] or J. Feldman and C. Moore [12]. In our setup :

Definition 16. Let $f: \mathbb{T} \rightarrow \mathbb{R}$ be measurable. An element $c \in \mathbb{R} \cup\{\infty\}$ is an essential value for $f$, if for any $\varepsilon>0$ and any Borel set $A \subset \mathbb{T}$ with $\lambda_{\mathbb{T}}(A)>0$, there is an $n \in \mathbb{Z}$ so that $\lambda_{\mathbb{T}}\left(A \cap T^{-n} A \cap\left\{\left|f_{n}-c\right|<\varepsilon\right\}\right)>0$.

We group in the next proposition standard results about essential values, which can be found for instance in $[\mathbf{2 2}]$.

Proposition 17. Denote by $\mathcal{E}(f)$ the set of finite essential values of $f$.

i) The set $\mathcal{E}(f)$ is a closed subgroup of $\mathbb{R}$.

ii) The skew-product $T_{f}$ on $\mathbb{T} \times \mathbb{R}$ is ergodic for $\lambda_{\mathbb{T}} \otimes \lambda_{\mathbb{R}}$ if and only if $\mathcal{E}(f)=\mathbb{R}$.

iii) Let the real measurable functions $f, g, h$ verify $f=g+h-T h$. Then the dynamical systems $\left(\mathbb{T} \times \mathbb{R}, T_{f}, \lambda_{\mathbb{T}} \otimes \lambda_{\mathbb{R}}\right)$ and $\left(\mathbb{T} \times \mathbb{R}, T_{g}, \lambda_{\mathbb{T}} \otimes \lambda_{\mathbb{R}}\right)$ are isomorphic.

A corollary of the last point is that when $f=h-T h$, then the dynamical system $\left(\mathbb{T} \times \mathbb{R}, T_{f}, \lambda_{\mathbb{T}} \otimes \lambda_{\mathbb{R}}\right)$ is not ergodic, because $\left(\mathbb{T} \times \mathbb{R}, T_{0}, \lambda_{\mathbb{T}} \otimes \lambda_{\mathbb{R}}\right)$ is not. Indeed, every function $(x, y) \longmapsto \psi(y)$ is $T_{0}$-invariant.

In order to show that $\mathcal{E}(f)=\mathbb{R}$, we will produce essential values via the following lemma. This is a particular case of Proposition 9 in M. Lemańczyk, F. Parreau and D. Volný [18] (see also [6] for a different proof). Recall that the vague topology on the space of signed 
Borel measures uses compactly supported continuous functions as test functions and that the set of non-negative Borel measures on $\mathbb{R}$ with mass not exceeding one is compact for the vague topology.

Lemma 18. If $k_{n} \rightarrow+\infty$ verifies $\left\|k_{n} \alpha\right\| \rightarrow 0$ and the law of $f_{k_{n}}$ (under $\lambda_{\mathbb{T}}$ ) converges to a measure $\nu$ for the vague topology, then $\operatorname{supp}(\nu) \subset \mathcal{E}(f)$.

We now start the proof of the second case of Theorem 4. First of all, since $f$ as in Lemma 15 is a continuous coboundary, we have

LEMma 19. Let $f$ be as in the statement of Lemma 15 and $\left(r_{n}\right)$ be a sequence of integers such that $\left\|r_{n} \alpha\right\| \rightarrow 0$. Then $\left\|f_{r_{n}}\right\|_{\infty} \rightarrow 0$.

Using this lemma, we recursively build functions $\left(f^{m}\right)_{m \geq 1}$, increasing sequences of integers $\left(N_{m}\right)_{m \geq 1},(\varphi(m))_{m \geq 1},\left(k_{m}\right)_{m \geq 1}$ and a sequence of reals $\left(B_{m}\right)_{m \geq 1}$. We still denote by $\left(p_{n} / q_{n}\right)$ the sequence of convergents of $\alpha$ and write $\left(a_{n}\right)$ for its partial quotients.

(i) When the $\left(a_{n}\right)$ are bounded. Let $f^{1}=0, N_{1}=0, B_{1}=0, k_{1}=0, \varphi(1)=5$. If $m>1$ and $f^{1}, \ldots, f^{m-1}$ are given, set $k_{m}=q_{\varphi(m)-4}$, where $\varphi(m)$ is chosen via Lemma 19 so that

$$
\left\|\sum_{l<m}\left(f^{l}\right)_{k_{m}}\right\|_{\infty}<1 / m
$$

Since for any $l$ we have $q_{l}=a_{l} q_{l-1}+q_{l-2}$ one can easily see that $k_{m} \leq q_{\varphi(m)} / 5$. Set $B_{m}=1 / k_{m}, N_{m}=\left\lfloor q_{\varphi(m)} / 4\right\rfloor$. We apply Lemma 15 with $\left(B_{m}, N_{m}\right)$ and $\Delta_{m}=1 /\left(8 q_{\varphi(m)}\right)$ and obtain $f^{m}$. We also impose on $\left(k_{m}\right)$ to verify

$$
\frac{k_{m^{\prime}}}{k_{m}}<2^{-m-m^{\prime}} \text { for } m^{\prime}<m \text { and } k_{m} \sum_{l>m} 1 / k_{l} \rightarrow_{m \rightarrow+\infty} 0 .
$$

(ii) When the $\left(a_{n}\right)$ are unbounded. Let $a_{\theta(n)} \rightarrow+\infty$ (with increasing $\left.(\theta(n))\right)$ and choose $r_{\theta(n)}=u_{\theta(n)} q_{\theta(n)-1}+q_{\theta(n)-2}$, with $1 \leq u_{\theta(n)} \leq a_{\theta(n)}$, so that $q_{\theta(n)} / 10 \leq r_{\theta(n)} \leq$ $q_{\theta(n)} / 5$, for large $n$. We have

$$
\left\|r_{\theta(n)} \alpha\right\| \leq a_{\theta(n)} / q_{\theta(n)}+1 / q_{\theta(n)-1} \leq 2 / q_{\theta(n)-1} \rightarrow_{n \rightarrow \infty} 0 .
$$

If $m>1$ and $f^{1}, \ldots, f^{m-1}$ have been chosen, by Lemma 19 take $k_{m}=r_{\theta(n(m))}$ so that (22) is verified, as well as (23). Set $\varphi(m)=\theta(n(m))$. Finally set $B_{m}=1 / k_{m}$, $N_{m}=\left\lfloor q_{\varphi(m)} / 4\right\rfloor$. We apply Lemma 15 with $\left(B_{m}, N_{m}\right)$ and $\Delta_{m}=1 /\left(8 q_{\varphi(m)}\right)$ and obtain a function $f^{m}$.

Remark that in both cases $k_{m} \leq q_{\varphi(m)} / 5$, from which we deduce that for large $m$

$$
N_{m}-k_{m} \geq q_{\varphi(m)} / 21
$$

In case (i) the boundedness of the $a_{n}$ 's implies that there is a constant $C^{\prime}>0$ such that for all $m$

$$
k_{m}=q_{\varphi(m)-4}>C^{\prime} q_{\varphi(m)},
$$


while in case (ii) taking $C^{\prime}=1 / 10$ we obtain

$$
k_{m}>q_{\varphi(m)} / 10=C^{\prime} q_{\varphi(m)} .
$$

Define now $f=\sum_{m \geq 1} f^{m}$. As $\sum_{m \geq 1} B_{m}<\infty, f$ is continuous. Fix $0 \leq \theta<1$. Recalling that $q_{m}$ grows at least exponentially fast for sufficiently large $m$ and for all $0 \leq \tau \leq 1$

$$
\frac{B_{m}}{\Delta_{m}}\left(\tau \Delta_{m}\right) \leq \frac{8 q_{\varphi(m)}}{k_{m}}\left(\tau \Delta_{m}\right)^{1-\theta}\left(\tau \Delta_{m}\right)^{\theta} \leq \frac{8}{C^{\prime}}\left(\Delta_{m}\right)^{1-\theta}\left(\tau \Delta_{m}\right)^{\theta} \leq \frac{1}{m^{2}}\left(\tau \Delta_{m}\right)^{\theta} .
$$

This implies again an inequality like (17). For $|x-y| \geq \Delta_{m}$ when $m$ is large one can again obtain (18).

We deduce as in Case i) of Theorem 4 that $f \in \cap_{0<\theta<1} C^{\theta}(\mathbb{T})$.

Condition (4) is true since by (14) each $f^{m}$ satisfies it.

It remains to check that the skew-product defined by $f$ is ergodic. We would like to apply Lemma 18. Consider the sequence $\left(k_{m}\right)$. By construction, $k_{m} \rightarrow+\infty$ and $\left\|k_{m} \alpha\right\| \rightarrow 0$. By compactness, after turning to a suitable subsequence, we can suppose that the law of $f_{k_{m}}$ on $(\mathbb{R}, \mathcal{B}(\mathbb{R}))$ converges for the vague topology to some non-negative Borel measure $\nu$ with mass not exceeding one. Next

$$
f_{k_{m}}(x)=\left(f^{m}\right)_{k_{m}}(x)+\sum_{l<m}\left(f^{l}\right)_{k_{m}}(x)+\sum_{l>m}\left(f^{l}\right)_{k_{m}}(x) .
$$

Due to (22), the second term on the right side uniformly goes to 0 . Due to (8), the last term is uniformly bounded by $k_{m} \sum_{l>m} 1 / k_{l} \rightarrow 0$, by $(23)$.

Consider now the intervals $\left[0, \Delta_{m}\right]+k \alpha$, for $0 \leq k<N_{m}-k_{m}$. For $x$ in any of these intervals, we have $\left(f^{m}\right)_{k_{m}}(x)=k_{m} f^{m}(x)$. Let $0 \leq a-\varepsilon<a<b<b+\varepsilon \leq 1$ and take a continuous function $\psi \geq 0$ with support in $[a-\varepsilon, b+\varepsilon]$, so that $\psi \geq 1$ on $[a-\varepsilon / 2, b+\varepsilon / 2]$. Using (24), we obtain

$$
\begin{aligned}
\int_{\mathbb{R}} \psi d \nu & =\lim _{m \rightarrow+\infty} \int_{\mathbb{T}} \psi\left(f_{k_{m}}\right) d \lambda_{\mathbb{T}} \\
& \geq \limsup _{m \rightarrow+\infty} \sum_{0 \leq k<N_{m}-k_{m}} \int_{k \alpha+(1-b) \Delta_{m}}^{k \alpha+(1-a) \Delta_{m}} \psi\left(f_{k_{m}}\right) d \lambda_{\mathbb{T}} \\
& \geq \limsup _{m \rightarrow+\infty}(b-a) \Delta_{m}\left(N_{m}-k_{m}\right) \geq \frac{(b-a)}{8 \cdot 21}>0
\end{aligned}
$$

As a result $[a-\varepsilon, b+\varepsilon] \cap \operatorname{supp}(\nu) \neq \emptyset$ and thus $[a-\varepsilon, b+\varepsilon] \cap \mathcal{E}(f) \neq \emptyset$ by Lemma 18 . The freedom on $a, b, \varepsilon$ implies that $\mathcal{E}(f)$ is dense in $[0,1]$. Since, by Proposition $17, \mathcal{E}(f)$ is an additive closed subgroup of $\mathbb{R}$, we have $\mathcal{E}(f)=\mathbb{R}$. Proposition 17 implies that the skew-product defined by $f$ is ergodic for $\lambda_{\mathbb{T}} \otimes \lambda_{\mathbb{R}}$. This concludes the proof of Case ii). 
Case iii). Fix $\varepsilon(n) \searrow 0$ as $n \nearrow+\infty$. Still writing $\left(q_{n}\right)$ for the denominators of the convergents of $\alpha$, choose an increasing sequence $(\varphi(m))_{m \geq 1}$ so that $q_{\varphi(m)}>4(1+m)$ and

$$
q_{\varphi(m)}^{1-1 / m} m \sum_{l=m+1}^{\infty} q_{\varphi(l)}^{-1+1 / l} \rightarrow 0, \quad q_{\varphi(m)}^{-1 / m} m \sum_{l=1}^{m-1} q_{\varphi(l)}^{1 / l} \rightarrow 0, \text { as } m \rightarrow+\infty .
$$

For $m \geq 1$, let $B_{m, 1}=q_{\varphi(m)}^{-1+1 / m}, N_{m, 1}=\left\lfloor q_{\varphi(m)} /(4(1+m))\right\rfloor$ and $\Delta_{m}=1 /\left(16 q_{\varphi(m)}\right)$. Set also $B_{m, 2}=B_{m, 1} / \sqrt{m}$ and $N_{m, 2}=m N_{m, 1}$. We can impose on $\varphi(m)$ to verify the additional condition

$$
q_{\varphi(m)}^{1 / m} \geq m^{2} \max \left\{\left(\left\lfloor N_{m, 1} / 2\right\rfloor\right)^{\varepsilon\left(\left\lfloor N_{m, 1} / 2\right\rfloor\right)},\left(\left\lfloor N_{m, 2} / 2\right\rfloor\right)^{\varepsilon\left(\left\lfloor N_{m, 2} / 2\right\rfloor\right)}\right\}
$$

Lemma 15 with $\left(B_{m, 1}, N_{m, 1}\right)$ and $\Delta_{m}$ gives $f^{m, 1}$ with support $F_{m, 1}$. Lemma 15 with $\left(B_{m, 2}, N_{m, 2}\right)$ and $\Delta_{m}$ furnishes $f^{m, 2, *}$ with support $F_{m, 2, *}$. Define then $f^{m, 2}(x)=$ $f^{m, 2, *}(x+1 / 2)$ and $F_{m, 2}=F_{m, 2, *}-1 / 2$.

A first observation is that $F_{m, 1} \cap F_{m, 2}=\emptyset$. Otherwise there are $k \neq l$ with $|k| \leq N_{m, 1},|l| \leq N_{m, 2}$ so that $|k \alpha-l \alpha+1 / 2| \leq 2 \Delta_{m}=1 /\left(8 q_{\varphi(m)}\right)$. This implies $\|2(k-l) \alpha\| \leq 1 /\left(4 q_{\varphi(m)}\right)$, whereas $2|k-l|<q_{\varphi(m)}$.

We now define

$$
\tilde{F}_{m, 1}=\bigcup_{0 \leq k<N_{m, 1} / 2}\left[-\Delta_{m} / 2, \Delta_{m} / 2\right]+k \alpha \subset F_{m, 1}
$$

and

$$
\tilde{F}_{m, 2}=\bigcup_{0 \leq k<N_{m, 2} / 2}\left[-\Delta_{m} / 2, \Delta_{m} / 2\right]+k \alpha \subset F_{m, 2}
$$

Clearly, for large $m$

$$
\lambda\left(\tilde{F}_{m, 1}\right) \geq 1 /(256 m) \text { and } \lambda\left(\tilde{F}_{m, 2}\right) \geq 1 / 256 .
$$

Because of the equidistribution of the sequence $(n \alpha)$, we can also assume that the strictly increasing sequence $\varphi(m)>m$ verifies the following property :

$$
\forall m^{\prime}, \forall m<m^{\prime}, \forall i, j \in\{1,2\}, 1-1 / m^{\prime} \leq \frac{\lambda\left(\tilde{F}_{m, i} \cap \tilde{F}_{m^{\prime}, j}\right)}{\lambda\left(\tilde{F}_{m, i}\right) \lambda\left(\tilde{F}_{m^{\prime}, j}\right)} \leq 1+1 / m^{\prime} .
$$

Define next

$$
f=\sum_{m \geq 1} \bar{f}^{m}, \text { with } \bar{f}^{m}=f^{m, 1}+f^{m, 2} .
$$

As $\sum_{m \geq 1}\left(B_{m, 1}+B_{m, 2}\right)<\infty$ the function $f$ is continuous. Fix again $0 \leq \theta<1$. One can easily see that $2 B_{m} / \Delta_{m}=32 q_{\varphi(m)}^{1 / m}$ is a Lipschitz constant for $\bar{f}^{m}$. Instead of (16) for large $m$ we have the following inequality

$$
32 q_{\varphi(m)}^{1 / m}\left(\tau \Delta_{m}\right) \leq 32 q_{\varphi(m)}^{1 / m}\left(\Delta_{m}\right)^{1-\theta}\left(\tau \Delta_{m}\right)^{\theta} \leq \frac{1}{m^{2}}\left(\tau \Delta_{m}\right)^{\theta}
$$

Prepared using etds.cls 
This again implies (17). For $|x-y|>\Delta_{m}$ instead of (18) we can use for large $m$

$$
\left|\bar{f}_{m}(x)-\bar{f}_{m}(y)\right| \leq 4 B_{m} \leq \frac{1}{m^{2}} \Delta_{m}^{\theta}<\frac{1}{m^{2}}|x-y|^{\theta} .
$$

We obtain $f \in \cap_{0<\theta<1} C^{\theta}(\mathbb{T})$. In order to prove $(5)$, let $x \in \mathbb{T}$. As $F_{m, 1} \cap F_{m, 2}=\emptyset$ for all $m \geq 1$, choose a sequence $\left(i_{m}\right)$ with $i_{m} \in\{1,2\}$ such that $x \notin F_{m, i_{m}}$ for all $m$. Because of (29) and (30), we have :

$$
\sum_{m \geq 1} \lambda\left(\tilde{F}_{m, i_{m}}\right)=+\infty \text { and } \liminf \frac{\sum_{m, m^{\prime}<N} \lambda\left(\tilde{F}_{m, i_{m}} \cap \tilde{F}_{m^{\prime}, i_{m^{\prime}}}\right)}{\left(\sum_{m<N} \lambda\left(\tilde{F}_{m, i_{m}}\right)\right)^{2}}=1
$$

Applying a generalization of the Borel-Cantelli lemma (cf A. Rényi [21], Lemma C, p. 391), we obtain that $\lambda\left(\lim \sup \tilde{F}_{m, i_{m}}\right)=1$. Take a point $y \in \lim \sup \tilde{F}_{m, i_{m}}$. Let then $m$ be arbitrary large such that $y \in \tilde{F}_{m, i_{m}}$. Set $P_{m, 1}=\left\lfloor N_{m, 1} / 2\right\rfloor$ and $P_{m, 2}=\left\lfloor N_{m, 2} / 2\right\rfloor$. We distinguish two cases :

1) Suppose that $i_{m}=1$. As $x \notin F_{m, 1}$, by $(13)$, we have $\left(f^{m, 1}\right)_{P_{m, 1}}(x) \leq 0$. Also $y \in \tilde{F}_{m, 1}$ implies $y \notin F_{m, 2}$ and $y+P_{m, 1} \alpha \in F_{m, 1}$ implies $y+P_{m, 1} \alpha \notin F_{m, 2}$, therefore by (12), $f_{P_{m, 1}}^{m, 2}(y)=0$. Thus, using (9) and (8)

$$
\begin{aligned}
f_{P_{m, 1}}(y)-f_{P_{m, 1}}(x) \geq & \bar{f}_{P_{m, 1}^{m}}^{m}(y)-f_{P_{m, 1}}^{m, 2}(x)+\sum_{l \geq 1, l \neq m}\left(\bar{f}_{P_{m, 1}}^{l}(y)-\bar{f}_{P_{m, 1}}^{l}(x)\right) \\
\geq & \frac{1}{3} N_{m, 1} B_{m, 1}(1 / 2-2 / \sqrt{m})-4 \sum_{1 \leq l<m} \sqrt{l} N_{l, 1} B_{l, 1}-4 N_{m, 1} \sum_{l>m} B_{l, 1} \\
& (\text { for large } m, \text { via }(25) \text { and }(26)) \\
\geq & \frac{N_{m, 1} B_{m, 1}}{7} \geq \frac{q_{\varphi(m)}^{1 / m}}{56 m} \geq \frac{m}{56}\left(P_{m, 1}\right)^{\varepsilon\left(P_{m, 1}\right)} .
\end{aligned}
$$

2) If $i_{m}=2$, then this time, by $(13),\left(f^{m, 2}\right)_{P_{m, 2}}(x) \leq 0$. As above we also have $f_{P_{m, 2}}^{m, 1}(y)=0$. Via $(9)$ and $(8)$

$$
\begin{aligned}
f_{P_{m, 2}}(y)-f_{P_{m, 2}}(x) & \geq \bar{f}_{P_{m, 2}}^{m}(y)-f_{P_{m, 2}}^{m, 1}(x)+\sum_{l \geq 1, l \neq m}\left(\bar{f}_{P_{m, 2}}^{l}(y)-\bar{f}_{P_{m, 2}}^{l}(x)\right) \\
& \geq \frac{1}{3} N_{m, 2} B_{m, 2}(1 / 2-2 / \sqrt{m})-4 \sum_{1 \leq l<m} N_{l, 2} B_{l, 2}-4 N_{m, 2} \sum_{l>m} B_{l, 1}
\end{aligned}
$$

(for large $m$ and when using (25) and (26))

$$
\geq \frac{N_{m, 2} B_{m, 2}}{7} \geq \frac{q_{\varphi(m)}^{1 / m}}{56 \sqrt{m}} \geq \frac{m^{3 / 2}}{56}\left(P_{m, 2}\right)^{\varepsilon\left(P_{m, 2}\right)}
$$

This finally shows (5), which ends the proof of Case iii) and of Theorem 4. 


\section{Smooth examples}

By taking advantage of the extra assumptions about the partial quotients of the rotation angle we now prove Theorem 10, with a different strategy. We still write $\left(a_{n}\right)$ for the partial quotients of $\alpha$ and $\left(p_{n} / q_{n}\right)$ for the sequence of convergents.

Recall that for $f \in C_{m 0}^{\infty}(\mathbb{T})$ the semi-norms $\left\|f^{(l)}\right\|_{\infty}$ form a non-decreasing function of $l \geq 0$. This will be used implicitly.

We fix for the whole section an even function $\psi \in C^{\infty}(\mathbb{R})$ verifying $\psi(x)=0$ for $x \notin[-1,1], \psi(x)>0$ for $x \in(-1,1), \psi^{\prime}(x)<0$ for $x \in(0,1)$ and $\int \psi=1$. Introduce the constants $A_{r}=\max _{1 \leq l \leq r}\left\|\psi^{(l)}\right\|_{L^{1}}, r \geq 1$.

For $B, \Delta>0$, introduce the linear peak $b_{B, \Delta}(x)=B \cdot(1-|x| / \Delta)_{+}$with support $[-\Delta, \Delta]$ as well as smooth versions:

$$
b_{B, \Delta, \delta}(x)=(\Delta \delta)^{-1} \int_{\mathbb{R}} b_{B, \Delta}(x-t) \psi(t /(\Delta \delta)) d t
$$

with support $[-\Delta(1+\delta), \Delta(1+\delta)]$. We have $\left\|b_{B, \Delta, \delta}^{(r)}\right\|_{\infty} \leq A_{r} B(\Delta \delta)^{-r}, r \geq 0$, and also $\left\|b_{B, \Delta, \delta}^{\prime}\right\|_{\infty} \leq B / \Delta$.

Proof of Theorem 10. Step 1. We first treat a common part of $i$ ) and $i i$ ). For $i$ ) define an increasing sequence $(\varphi(m))_{m \geq 1}$ so that

$$
\left\{\begin{array}{c}
a_{\varphi(m)+1} \geq 4^{r+1} A_{r+1} m^{3 r+6} e^{(r+1) \sqrt{m}} q_{\varphi(m)}^{r-1}, \text { for the } C^{r} \text { case } \\
a_{\varphi(m)+1} \geq 4^{m+1} A_{m+1} m^{3 m+6} e^{(m+1) \sqrt{m}} q_{\varphi(m)}^{m-1}, \text { for the } C^{\infty} \text { case }
\end{array}\right.
$$

and define parameters

$$
B_{m}=m / q_{\varphi(m)}, \quad \Delta_{m}=e^{-\sqrt{m}} /\left(4 q_{\varphi(m)}\right), \quad \delta_{m}=m^{-3}
$$

Concerning $i$ ) impose on $\varphi$ the following conditions

$$
\left\{\begin{array}{c}
a_{\varphi(m)+1} \geq 8^{r+1} A_{r+1} m^{3 r+5} q_{\varphi(m)}^{r-1}, \text { for the } C^{r} \text { case } \\
a_{\varphi(m)+1} \geq 8^{m+1} A_{m+1} m^{3 m+5} q_{\varphi(m)}^{m-1}, \text { for the } C^{\infty} \text { case }
\end{array}\right.
$$

and let

$$
B_{m}=1 / q_{\varphi(m)}, \quad \Delta_{m}=1 /\left(8 q_{\varphi(m)}\right), \quad \delta_{m}=m^{-3} .
$$

Now for both cases $i$ ) and $i i$ ) fix $m \geq 1$ and let $h^{m}=b_{B_{m}, \Delta_{m}, \delta_{m}}$ and also

$$
f^{m}=\sum_{k=0}^{q_{\varphi(m)}-1} T^{-k}\left(h^{m}-T^{q_{\varphi(m)}} h^{m}\right) .
$$


By our assumptions about $a_{\varphi(m)+1}$ the value of $q_{\varphi(m)+1}=a_{\varphi(m)+1} q_{\varphi(m)}+q_{\varphi(m)-1} \gg q_{\varphi(m)}$ and the points $0, \alpha, \ldots,\left(q_{\varphi(m)}-1\right) \alpha$ are "almost equally" spaced on $\mathbb{T}$. The supports of $h^{m}$ and $T^{q_{\varphi(m)}} h^{m}$ almost completely overlap and the $T^{-k}\left(h^{m}-T^{q_{\varphi(m)}} h^{m}\right), 0 \leq k<q_{\varphi(m)}$, have disjoint supports for large $m$. Also $f^{m}$ is a coboundary $f^{m}=H^{m}-T H^{m}$, with $H^{m}=K^{m}+L^{m}$ where $K^{m}$ and $L^{m}$ are given by

$$
K^{m}=q_{\varphi(m)} \sum_{k=0}^{q_{\varphi(m)}-1} T^{k} h^{m}, L^{m}=\sum_{l=1}^{q_{\varphi(m)}-1}\left(q_{\varphi(m)}-l\right) T^{-l}\left(h^{m}-T^{q_{\varphi(m)}} h^{m}\right) .
$$

By definition, $h^{m} \in C^{\infty}(\mathbb{T})$. For $r^{\prime} \geq 0$, we have

$$
\begin{aligned}
\left\|\left(h^{m}\right)^{\left(r^{\prime}\right)}-T^{q_{\varphi(m)}}\left(h^{m}\right)^{\left(r^{\prime}\right)}\right\|_{\infty} & \leq\left\|q_{\varphi(m)} \alpha\right\|\left\|\left(h^{m}\right)^{\left(r^{\prime}+1\right)}\right\|_{\infty} \\
& \leq A_{r^{\prime}+1} \frac{B_{m}\left(\Delta_{m} \delta_{m}\right)^{-r^{\prime}-1}}{a_{\varphi(m)+1} q_{\varphi(m)}} .
\end{aligned}
$$

Using (34) and (36) with $r^{\prime}=r$, or $r^{\prime}=m$ we get

$$
\left\|\left(f^{m}\right)^{(r)}\right\|_{\infty} \leq 1 / m^{2}\left(C^{r} \text {-case }\right),\left\|\left(f^{m}\right)^{(m)}\right\|_{\infty} \leq 1 / m^{2}\left(C^{\infty} \text {-case }\right) .
$$

From (36) with $r^{\prime}=0 \leq r-1$ and the definition of $\varphi(m)$, one deduces from (35) and from the disjointness of the supports of $T^{-k}\left(h^{m}-T^{q_{\varphi(m)}} h^{m}\right)$ that

$$
\left\|L^{m}\right\|_{\infty} \leq 1 / m^{2}
$$

Consider next the shortfall of maximization (cf. (2)) of the point 0 for $f^{m}$. Via (38) we have

$$
S M\left(f^{m}, 0\right) \leq S M\left(K^{m}-T K^{m}, 0\right)+4 / m^{2} .
$$

We turn to $S M\left(K^{m}-T K^{m}, 0\right)$. Using $\left(K^{m}-T K^{m}\right)_{n}=K^{m}-T^{n} K^{m}$, the density of $\left(T^{m} 0\right)$ and continuity of $K^{m}$ we obtain with $\xi^{m}(x, y)=K^{m}(x)-K^{m}(x+y)-K^{m}(0)+K^{m}(y)$ that

$$
S M\left(K^{m}-T K^{m}, 0\right)=\sup _{x, y \in \mathbb{T}} \xi^{m}(x, y)
$$

By definition of $h^{m}$ and $K^{m}$ we have $K^{m} \geq 0$ and $K^{m}(0) \geq K^{m}(y)$ for all $y \in \mathbb{T}$. Also $h^{m}$ is even and $\left(h^{m}\right)^{\prime}$ is negative on $\left(0, \Delta_{m}\left(1+\delta_{m}\right)\right)$, decreasing on $\left[0, \delta_{m} \Delta_{m}\right]$, equals $-B_{m} / \Delta_{m}$ on $\left[\delta_{m} \Delta_{m}, \Delta_{m}\left(1-\delta_{m}\right)\right]$, increasing to zero on $\left[\Delta_{m}\left(1-\delta_{m}\right), \Delta_{m}\left(1+\delta_{m}\right)\right]$.

Fix now $y$ and take $x$ maximizing $K^{m}(x)-K^{m}(x+y)$. We can assume $K^{m}(x)>K^{m}(x+y)$, because otherwise $\xi^{m}(x, y) \leq 0$ whereas $S M\left(K^{m}-T K^{m}, 0\right) \geq 0$ by (40). In particular, $K^{m}(x)>0$ and for some $0 \leq k<q_{\varphi(m)}$ we have $x \in-k \alpha+\left[-\Delta_{m}\left(1+\delta_{m}\right), \Delta_{m}\left(1+\delta_{m}\right)\right]$. 
If $x \in-k \alpha+\left(\left[-\Delta_{m}\left(1+\delta_{m}\right),-\Delta_{m}\left(1-\delta_{m}\right)\right] \cup\left[\Delta_{m}\left(1-\delta_{m}\right), \Delta_{m}\left(1+\delta_{m}\right)\right]\right)$ then

$$
\xi^{m}(x, y) \leq K^{m}(x) \leq 2 q_{\varphi(m)} \delta_{m} \Delta_{m} \cdot \frac{B_{m}}{\Delta_{m}} \leq 2 / m^{2} .
$$

Next we suppose that $x \in-k \alpha+\left[-\Delta_{m}\left(1-\delta_{m}\right), \Delta_{m}\left(1-\delta_{m}\right)\right]$. If $x \notin-k \alpha+$ $\left[-\delta_{m} \Delta_{m}, \delta_{m} \Delta_{m}\right]$, observe that $B_{m} / \Delta_{m}=\left|\left(K^{m}\right)^{\prime}(x)\right| \geq\left|\left(K^{m}\right)^{\prime}(x+y)\right|$ and the distance between $x+y$ and any $-l \alpha, 0 \leq l<q_{\varphi(m)}$, is at least $|-k \alpha-x|$ since we assumed $K^{m}(x)>K^{m}(x+y)$. When moving $x$ towards $-k \alpha$, the quantity $K^{m}(x)-K^{m}(x+y)$ does not decrease until $x$ reaches $-k \alpha \pm \delta_{m} \Delta_{m}$. As a result, one can always assume that $x \in-k \alpha+\left[-\delta_{m} \Delta_{m}, \delta_{m} \Delta_{m}\right]$. Writing $x=-k \alpha+s$, with $|s| \leq \delta_{m} \Delta_{m}$ we have

$$
\begin{aligned}
\xi^{m}(x, y) & \leq K^{m}(y)-K^{m}(x+y) \\
& \leq\left|K^{m}(y)-K^{m}(y+s)\right|+\left|K^{m}(y+s)-K^{m}(y+s-k \alpha)\right|
\end{aligned}
$$

For the first term on the right-hand side, we have

$$
\left|K^{m}(y)-K^{m}(y+s)\right| \leq q_{\varphi(m)}|s|\left\|\left(h^{m}\right)^{\prime}\right\|_{\infty} \leq q_{\varphi(m)} \delta_{m} \Delta_{m} \cdot \frac{B_{m}}{\Delta_{m}} \leq m^{-2}
$$

For the second term write $y+s=-l \alpha+t$, with $0 \leq l<q_{\varphi(m)}$ and $|t|<1 / q_{\varphi(m)}$. Since $K^{m}$ is nearly " $\alpha$-periodic", we have

$$
\begin{aligned}
\left|K^{m}(-l \alpha+t)-K^{m}(-(k+l) \alpha+t)\right| & \leq q_{\varphi(m)}\left\|q_{\varphi(m)} \alpha\right\|\left\|\left(h^{m}\right)^{\prime}\right\|_{\infty} \\
& \leq a_{\varphi(m)+1}^{-1} \cdot B_{m} / \Delta_{m} \\
& \leq 1 / m^{2}
\end{aligned}
$$

From (41-44) we obtain $\xi^{m}(x, y) \leq 2 / m^{2}$. Using now (39) and (40)

$$
S M\left(f^{m}, 0\right) \leq 6 / m^{2}
$$

We can now set $f=\sum_{m \geq 1} f^{m}$. By (37) and the monotonicity of the semi-norms, $f \in C_{m 0}^{r}(\mathbb{T})$ in the $C^{r}$-case and $f \in C_{m 0}^{\infty}(\mathbb{T})$ in the $C^{\infty}$-case. Using $\sum_{m \geq 1} 1 / m^{2} \leq 2$ and (45) we obtain

$$
\forall n \geq 0, \forall x \in \mathbb{T}, \quad f_{n}(x) \leq f_{n}(0)+12 .
$$

As a result 0 is a maximizing point for $f$.

Step 2. We complete the proof of $i$ ). First, since $H^{m}=K^{m}+L^{m}$, we deduce from (35) and (38) that $\left\|H^{m}\right\|_{\infty} \geq m / 2$ for large $m$. Since $f^{m}=H^{m}-T H^{m}$ and $H^{m}(x)=0$ for 
some $x \in \mathbb{T}$ we get $\sup _{n \geq 0}\left\|\left(f^{m}\right)_{n}\right\|_{\infty} \geq\left\|H^{m}\right\|_{\infty} \geq m / 2$, for large $m$. Also, using (45) and since $\left(f^{m}\right)_{n}(x)=0$ for some $x \in \mathbb{T}$, we get $\left(f^{m}\right)_{n}(0) \geq-6 / m^{2}$. For any integer $m \geq 1$, we obtain via (45)

$$
\begin{aligned}
\sup _{n \geq 0} f_{n}(0) & \geq \sup _{n \geq 0}\left(f^{m}\right)_{n}(0)-6 \sum_{l \neq m} 1 / l^{2} \\
& \geq \sup _{n \geq 0}\left\|\left(f^{m}\right)_{n}\right\|_{\infty}-6 \sum_{l} 1 / l^{2} \geq m / 2-12,
\end{aligned}
$$

for arbitrary large $m$. We deduce that $\sup _{n \geq 0} f_{n}(0)=+\infty$, which implies that $f$ is not a continuous coboundary.

We now show that $f=g-T g$, for some $g \in \cap_{1<p<\infty} L^{p}(\mathbb{T})$. Let $m \geq 1$. The support $U_{m}$ of $H^{m}$ has measure less than $e^{-\sqrt{m}} / 2$. Let $N \geq 1$ and $V_{N}=\cup_{m>N} U_{m}$. If both $x$ and $T^{n} x$ lie outside $V_{N}$, then $\left|f_{n}(x)\right| \leq \sum_{1 \leq m \leq N} m \leq N^{2}$. Hence for all $n \geq 0$ and $N \geq 1$

$$
\lambda\left\{x \in \mathbb{T}:\left|f_{n}(x)\right| \leq N^{2}\right\} \geq 1-\sum_{m>N} e^{-\sqrt{m}} .
$$

From this we infer as in Theorem 4 that $\left(f_{n}\right)$ is bounded in $L^{p}(\mathbb{T})$ for all $1 \leq p<\infty$. Via [19] this concludes the proof of $i$ ).

We next complete the proof of $i i)$. Let $k_{m}=\left\lfloor a_{\varphi(m)+1} / 3\right\rfloor q_{\varphi(m)}$. We will use the property that $2 \Delta_{m}<\left\|k_{m} \alpha\right\|<5 \Delta_{m}$ and hence $K^{m}$ and $T^{k_{m}} K^{m}$ have disjoint supports. One can impose on $\varphi$ to verify also the following conditions

$$
\left\|\sum_{l<m}\left(f^{l}\right)_{k_{m}}\right\|_{\infty} \leq 1 / m, k_{m} \sum_{l>m} 1 / q_{\varphi(l)} \leq 1 / m .
$$

Remark that $\left\|k_{m} \alpha\right\| \leq 2 / q_{\varphi(m)} \rightarrow 0$. We wish to apply Lemma 18 with the sequence $\left(k_{m}\right)$. We have

$$
f_{k_{m}}=\sum_{l<m}\left(f^{l}\right)_{k_{m}}+\left(f^{m}\right)_{k_{m}}+\sum_{l>m}\left(f^{l}\right)_{k_{m}}
$$

Using (46), the first and third terms on the right-hand side are both bounded by $1 / \mathrm{m}$. Consider the middle term

$$
\left(f^{m}\right)_{k_{m}}=K^{m}-T^{k_{m}} K^{m}+L^{m}-T^{k_{m}} L^{m}
$$

By (38), $\left\|L^{m}\right\|_{\infty} \leq 1 / m^{2}$, so we focus on $K^{m}-T^{k_{m}} K^{m}$.

Recall that $K^{m}=\sum_{k=0}^{q_{\varphi(m)}-1} q_{\varphi(m)} T^{k} h^{m} ;$ moreover $K^{m}$ and $T^{k_{m}} K^{m}$ have disjoint supports. The maximum of $q_{\varphi(m)} h^{m}$ tends to one. Let $1 / 4<a<b<3 / 4$. Take $\varepsilon>0$ small enough and a continuous function $\kappa(x) \geq 0$ with support $[a, b]$ so that 
$\kappa(x) \geq 1$ for $x \in(a+\varepsilon, b-\varepsilon)$. Also $q_{\varphi(m)} h^{m}$ is linear on $\left[-\Delta_{m}\left(1-\delta_{m}\right),-\delta_{m} \Delta_{m}\right]$ and on $\left[\Delta_{m} \delta_{m},\left(1-\delta_{m}\right) \Delta_{m}\right]$ and the absolute value of the slope is between $1 /\left(2 \Delta_{m}\right)$ and $2 / \Delta_{m}$.

Up to considering a subsequence, suppose that the law of $f_{k_{m}}$ converges to a measure $\nu$ for the vague topology. Then

$$
\begin{aligned}
\int_{\mathbb{R}} \kappa d \nu & =\lim _{m \rightarrow+\infty} \int_{\mathbb{T}} \kappa\left(f_{k_{m}}\right) d \lambda_{\mathbb{T}}=\lim _{m \rightarrow+\infty} \int_{\mathbb{T}} \kappa\left(K^{m}-T^{k_{m}} K^{m}\right) d \lambda_{\mathbb{T}} \\
& \geq \limsup _{m \rightarrow+\infty} \sum_{0 \leq k \leq q_{\varphi(m)-1}} \int_{\mathbb{T}} \kappa\left(q_{\varphi(m)} T^{k} h^{m}\right) d \lambda_{\mathbb{T}} \\
& \geq \limsup _{m \rightarrow+\infty} q_{\varphi(m)} \int_{\mathbb{T}} \kappa\left(q_{\varphi(m)} h^{m}\right) d \lambda_{\mathbb{T}} \geq q_{\varphi(m)} \frac{(b-a)}{2 / \Delta_{m}}=\frac{(b-a)}{16}>0
\end{aligned}
$$

As a result $\operatorname{supp}(\nu)$ intersects $[a, b]$ and we conclude that $[1 / 4,3 / 4] \subset \operatorname{supp}(\nu)$. Lemma 18 then says that $\mathcal{E}(f)=\mathbb{R}$. By Proposition 17 , the skew-product defined by $f$ is ergodic. This concludes the proof of $i i)$.

Step 3. We turn to $i i i)$. Define an increasing sequence $(\varphi(m))_{m \geq 1}$ so that

$$
\left\{\begin{array}{c}
a_{\varphi(m)+1} \geq 8^{r+1} A_{r+1} m^{4 r+7} q_{\varphi(m)}^{r-1}, \text { for the } C^{r} \text { case } \\
a_{\varphi(m)+1} \geq 8^{m+1} A_{m+1} m^{4 m+7} q_{\varphi(m)}^{m-1}, \text { for the } C^{\infty} \text { case }
\end{array}\right.
$$

and introduce parameters

$$
B_{m}=\sqrt{m} / q_{\varphi(m)}, \quad \Delta_{m}=1 /\left(8 m q_{\varphi(m)}\right), \quad \delta_{m}=m^{-3}
$$

Set also $B_{m}^{\prime}=\sqrt{m} B_{m}$ and $\Delta_{m}^{\prime}=m \Delta_{m}$. Let $h^{m}=b_{B_{m}, \Delta_{m}, \delta_{m}}$ and $\tilde{h}^{m}=b_{B_{m}^{\prime}, \Delta_{m}^{\prime}, \delta_{m}}(.-$ $\left.x_{m}\right)$, where $x_{m}=1 /\left(2 q_{\varphi(m)}\right)$.

Put $f^{m}$ as in (34) and $\tilde{f}^{m}$ similarly via $\tilde{h}^{m}$. We have $f^{m}=H^{m}-T H^{m}$, with $H^{m}=K^{m}+L^{m}$, and $\tilde{f}^{m}=\tilde{H}^{m}-T \tilde{H}^{m}$, with $\tilde{H}^{m}=\tilde{K}^{m}+\tilde{L}^{m}$, as in (35).

Using as before (34) and (36) with $r^{\prime}=r$ or $r^{\prime}=m$ we get (37) for both $f^{m}$ and $\tilde{f}^{m}$. Setting $f=\sum_{m \geq 1} g^{m}$, with $g^{m}=f^{m}+\tilde{f}^{m}$, we deduce that $f \in C_{m 0}^{r}(\mathbb{T})$ in the $C^{r}$-case and that $f \in C_{m 0}^{\infty}(\mathbb{T})$ in the $C^{\infty}$-case.

We now check that $f$ has no maximizing points. This will require some further assumptions on $\varphi$. Let $b_{m}=\left\lfloor a_{\varphi(m)+1} /(16 m)\right\rfloor$ and $N_{m}=b_{m} q_{\varphi(m)}$ so that $\left\|N_{m} \alpha\right\| \epsilon$ $\left[1 /\left(20 m q_{\varphi(m)}\right), 1 /\left(10 m q_{\varphi(m)}\right)\right]$ for large $m$. Then

$$
\begin{aligned}
f_{N_{m}}(0)-f_{N_{m}}(x) & =\sum_{1 \leq l<m}\left(\left(g^{l}\right)_{N_{m}}(0)-\left(g^{l}\right)_{N_{m}}(x)\right) \\
& +\left(g^{m}\right)_{N_{m}}(0)-\left(g^{m}\right)_{N_{m}}(x)+\sum_{l>m}\left(\left(g^{l}\right)_{N_{m}}(0)-\left(g^{l}\right)_{N_{m}}(x)\right) .
\end{aligned}
$$

Prepared using etds.cls 
As $\left\|N_{m} \alpha\right\| \leq 1 / q_{\varphi(m)}$, one can choose $\varphi$ so that $\left\|\sum_{l<m}\left(g^{l}\right)_{N_{m}}\right\|_{\infty} \rightarrow 0$ and in this case the first term on the right-hand side uniformly goes to 0 . The third term is bounded by $2 N_{m} \sum_{l>m}\left\|q_{\varphi(l)} \alpha\right\| \max \left\{B_{l} / \Delta_{l}, B_{l}^{\prime} / \Delta_{l}^{\prime}\right\} \leq 2 N_{m} \sum_{l>m} 1 / q_{\varphi(l)}$ and $\varphi$ can be chosen so that this uniformly goes to 0 .

It remains to focus on the middle term. Remark that (38) is valid for both $L^{m}$ and $\tilde{L}^{m}$. We first have, using that $0 \notin \operatorname{supp}\left(\tilde{K}^{m}\right) \cup \operatorname{supp}\left(T^{N_{m}} \tilde{K}^{m}\right)$ :

$$
\begin{aligned}
\left(g^{m}\right)_{N_{m}}(0) & =H^{m}(0)-T^{N_{m}} H^{m}(0)+\left(\tilde{H}^{m}(0)-T^{N_{m}} \tilde{H}^{m}(0)\right) \\
& \geq K^{m}(0)-T^{N_{m}} K^{m}(0)+\left(\tilde{K}^{m}(0)-T^{N_{m}} \tilde{K}^{m}(0)\right)-4 / m^{2} \\
& \geq K^{m}(0)-T^{N_{m}} K^{m}(0)-4 / m^{2} \\
& \geq\left\|N_{m} \alpha\right\|\left(q_{\varphi(m)} B_{m} /\left(2 \Delta_{m}\right)\right)-4 / m^{2} \geq \sqrt{m} / 5-4 / m^{2} .
\end{aligned}
$$

Making now the hypothesis that $x \notin \operatorname{supp}\left(K^{m}\right) \cup \operatorname{supp}\left(T^{N_{m}} K^{m}\right)$, we have

$$
\begin{aligned}
\left(g^{m}\right)_{N_{m}}(x) & \leq \tilde{K}^{m}(x)-T^{N_{m}} \tilde{K}^{m}(x)+4 / m^{2} \\
& \leq\left\|N_{m} \alpha\right\|\left(q_{\varphi(m)} 2 B_{m}^{\prime} / \Delta_{m}^{\prime}\right)+4 / m^{2} \leq 8 / 5+4 / m^{2} .
\end{aligned}
$$

As a result, the previous discussion entails that for large $m$ and if $x \notin \operatorname{supp}\left(K^{m}\right) \cup$ $\operatorname{supp}\left(T^{N_{m}} K^{m}\right)$, then $f_{N_{m}}(0)-f_{N_{m}}(x) \geq \sqrt{m} / 10$.

In a symmetric way, define $b_{m}^{\prime}=\left\lfloor a_{\varphi(m)+1} / 7\right\rfloor$ and $N_{m}^{\prime}=b_{m}^{\prime} q_{\varphi(m)}$. We consider this time

$$
\begin{aligned}
f_{N_{m}^{\prime}}\left(x_{m}\right)-f_{N_{m}^{\prime}}(x) & =\sum_{1 \leq l<m}\left(\left(g^{l}\right)_{N_{m}^{\prime}}\left(x_{m}\right)-\left(g^{l}\right)_{N_{m}^{\prime}}(x)\right) \\
& +\left(g^{m}\right)_{N_{m}^{\prime}}\left(x_{m}\right)-\left(g^{m}\right)_{N_{m}^{\prime}}(x)+\sum_{l>m}\left(\left(g^{l}\right)_{N_{m}^{\prime}}\left(x_{m}\right)-\left(g^{l}\right)_{N_{m}^{\prime}}(x)\right) .
\end{aligned}
$$

Since $\left\|N_{m}^{\prime} \alpha\right\| \leq 1 / q_{\varphi(m)}$ and the third term is bounded by $2 N_{m}^{\prime} \sum_{l>m} 1 / q_{\varphi(l)}$, as above $\varphi$ can be chosen so that the first and third terms uniformly go to 0 . We now have, using that $x_{m} \notin \operatorname{supp}\left(K^{m}\right) \cup \operatorname{supp}\left(T^{N_{m}^{\prime}} K^{m}\right) \cup \operatorname{supp}\left(T^{N_{m}^{\prime}} \tilde{K}^{m}\right)$ :

$$
\begin{aligned}
\left(g^{m}\right)_{N_{m}^{\prime}}\left(x_{m}\right) & =H^{m}\left(x_{m}\right)-T^{N_{m}^{\prime}} H^{m}\left(x_{m}\right)+\left(\tilde{H}^{m}\left(x_{m}\right)-T^{N_{m}^{\prime}} \tilde{H}^{m}\left(x_{m}\right)\right) \\
& \geq K^{m}\left(x_{m}\right)-T^{N_{m}^{\prime}} K^{m}\left(x_{m}\right)+\left(\tilde{K}^{m}\left(x_{m}\right)-T^{N_{m}^{\prime}} \tilde{K}^{m}\left(x_{m}\right)\right)-4 / m^{2} \\
& \geq\left(\tilde{K}^{m}\left(x_{m}\right)-T^{N_{m}^{\prime}} \tilde{K}^{m}\left(x_{m}\right)\right)-4 / m^{2} \geq m / 2-4 / m^{2} .
\end{aligned}
$$

Supposing now that $x \notin \operatorname{supp}\left(\tilde{K}^{m}\right) \cup \operatorname{supp}\left(T^{N_{m}^{\prime}} \tilde{K}^{m}\right)$, we get

$$
\left(g^{m}\right)_{N_{m}^{\prime}}(x) \leq K^{m}(x)-T^{N_{m}} K^{m}(x)+4 / m^{2} \leq 2 \sqrt{m}+4 / m^{2} .
$$

As a consequence of the preceding inequalities, for large $m$ and if $x \notin \operatorname{supp}\left(\tilde{K}^{m}\right) \cup$ $\operatorname{supp}\left(T^{N_{m}^{\prime}} \tilde{K}^{m}\right)$, then $f_{N_{m}^{\prime}}\left(x_{m}\right)-f_{N_{m}^{\prime}}(x) \geq m / 4$.

To conclude, we only have to remark that $\operatorname{supp}\left(K^{m}\right) \cup \operatorname{supp}\left(T^{N_{m}} K^{m}\right)$ and $\operatorname{supp}\left(\tilde{K}^{m}\right) \cup$ $\operatorname{supp}\left(T^{N_{m}^{\prime}} \tilde{K}^{m}\right)$ are disjoint. This completes the proof of iii) and the theorem. 
6. Generic behaviour

6.1. Rotations. Recall that $\{c+g-T g, c \in \mathbb{R}, g \in C(\mathbb{T})\}$ is dense in $C(\mathbb{T})$, for instance since trigonometric polynomials with zero constant term are $C(\mathbb{T})$-coboundaries and are dense in $C_{m 0}(\mathbb{T})$.

Proof of Theorem 13. Choose $c_{l} \in \mathbb{Q}$ and $g^{l} \in C(\mathbb{T})$ such that $\left\{c_{l}+g^{l}-T g^{l}\right\}_{l=1}^{\infty}$ is dense in $C(\mathbb{T})$.

Given $n$ we will select a dense open set $\mathcal{G}_{n}$ in $C(\mathbb{T})$, using the functions defined during the proof of Case iii) of Theorem 4 in (31) as perturbation functions. We denote these functions by $f^{l, n}$ with parameters $B_{m,(l, n)}, B_{m,(l, n)}^{\prime}$ and $N_{m,(l, n)}, N_{m,(l, n)}^{\prime}$ appearing in their construction. By a suitable choice of the bounds $B_{m,(l, n)}$ and $B_{m,(l, n)}^{\prime}$ we can always achieve that $\left\|f^{l, n}\right\|_{\infty}<1$.

Take now $m(l, n)$ so large that $N_{m(l, n),(l, n)}, N_{m(l, n),(l, n)}^{\prime}>n,(32)$ and (33) are both applicable and (using that $q_{\varphi(m)}^{1 / m} / m \rightarrow+\infty$, by $(25)$ )

$$
\frac{q_{\varphi(m(l, n))}^{1 / m(l, n)}}{16 n m(l, n)}-4\left\|g^{l}\right\|_{\infty}>n .
$$

Set $\tilde{f}^{l, n}=c_{l}+g^{l}-T g^{l}+f^{l, n} / n$. Then by (32), (33) and (47) for any $x \in \mathbb{T}$

$$
\begin{gathered}
\max \left\{\tilde{f}_{N_{m(l, n)}, n}^{l, n}(0)-\tilde{f}_{N_{m(l, n)}^{l, n}}^{l}(x), \tilde{f}_{N_{m(l, n)}^{\prime}}^{l, n}(1 / 2)-\tilde{f}_{N_{m(l, n)}^{\prime}}^{l, n}(x)\right\} \\
\geq \frac{q_{\varphi(m(l, n))}^{1 / m(l, n)}}{16 n m(l, n)}-4\left\|g^{l}\right\|_{\infty}>n
\end{gathered}
$$

Since $N_{m(l, n)}$ and $N_{m(l, n)}^{\prime}$ are fixed numbers one can choose $\varepsilon_{l, n}>0$ such that for all $f \in B\left(\tilde{f}^{l, n}, \varepsilon_{l, n}\right)$ we have for all $x \in \mathbb{T}$

$$
\max \left\{f_{N_{m(l, n)}}(0)-f_{N_{m(l, n)}}(x), f_{N_{m(l, n)}^{\prime}}(1 / 2)-f_{N_{m(l, n)^{\prime}}}(x)\right\} \geq n-1 .
$$

Set

$$
\mathcal{G}_{n}=\cup_{l=1}^{\infty} B\left(\tilde{f}^{l, n}, \varepsilon_{l, n}\right) \text { and } \mathcal{G}=\cap_{n=1}^{\infty} \mathcal{G}_{n}
$$

Clearly, $\mathcal{G}_{n}$ is dense and open in $C(\mathbb{T})$ and if $f \in \mathcal{G}$ then for any $n$ there exists $l_{n}$ such that we have (48) with $l_{n}$ instead of $l$. Therefore $f$ does not have a weakly maximizing point.

6.2. The transformation $\mathbf{T} \mathbf{x}=\mathbf{2} \mathbf{x} \bmod (\mathbf{1}) \quad$ A modification of the above methods can be applied for the transformation $T x=2 x \bmod (1)$ on $\mathbb{T}$. It is a significant difference that the continuous functions of the form $c+g-T g$ do not give us a dense subset in $C(\mathbb{T})$. We denote by $\mathcal{M}$ the set of $T$-invariant Borel probability measures on $\mathbb{T}$. A function $f \in C(\mathbb{T})$ is called a weak coboundary if $\int f d \mu=0$ for all $\mu \in \mathcal{M}$. Lemma 3 of [5] states that $f \in C(\mathbb{T})$ is a weak coboundary if and only if it is a uniform limit of coboundaries. The set of weak coboundaries is nowhere dense and closed in $C(\mathbb{T})$, while coboundaries form a non-closed proper subset of weak coboundaries (see [5]). 
We select a dense set $\left\{h^{l}\right\}_{l=1}^{\infty}$ in $C(\mathbb{T})$ which consists of Lipschitz continuous functions, that is

$$
\left|h^{l}(x)-h^{l}\left(x^{\prime}\right)\right| \leq L_{l}\left|x-x^{\prime}\right| \text { for all } x, x^{\prime} \in \mathbb{T} .
$$

As it was mentioned in the introduction each $h^{l}$ has maximizing points. Choose and denote one such point by $z_{l}$. This means that

$$
\forall x \in \mathbb{T}, \forall n \in \mathbb{N} \text { we have } h_{n}^{l}(x) \leq h_{n}^{l}\left(z_{l}\right)+C_{l} .
$$

We will use the dense set $\left\{h_{l}\right\}_{l=1}^{\infty}$ to prove Theorem 14. The perturbation functions used in the proof of this theorem are given by the following lemma:

Lemma 20. Suppose $z \in \mathbb{T}, m \in \mathbb{N}$ are given. There exist $\bar{f}^{m} \in C(\mathbb{T}), x_{m}, y_{m} \in \mathbb{T}$ such that if $N_{m}=2 m^{2}, N_{m}^{\prime}=200 m^{2}=100 N_{m}$ then

$$
\begin{gathered}
\left\|\bar{f}^{m}\right\|_{\infty}<1, \quad\left|x_{m}-z\right|<2^{-N_{m}}, \quad\left|y_{m}-z\right|<2^{-N_{m}^{\prime}}, \\
\forall x \in \mathbb{T}, \quad \max \left\{\bar{f}_{N_{m}}^{m}\left(x_{m}\right)-\bar{f}_{N_{m}}^{m}(x), \bar{f}_{N_{m}^{\prime}}^{m}\left(y_{m}\right)-\bar{f}_{N_{m}^{\prime}}^{m}(x)\right\}>m^{2} .
\end{gathered}
$$

Proof. Let $\bar{f}_{u, \Delta, B}(x)=B(1-|u-x| / \Delta)_{+}, B_{m}=1 / 2, B_{m}^{\prime}=B_{m} / 10=1 / 20$. Denote by $\mathcal{S}_{u, m}$ (resp. $\left.\mathcal{S}_{u, m}^{\prime}\right)$ the support of $\bar{f}_{u, \Delta_{m}, B_{m}}\left(\right.$ resp. $\left.\bar{f}_{u, \Delta_{m}^{\prime}, B_{m}^{\prime}}\right)$, where $\Delta_{m}, \Delta_{m}^{\prime}$ are chosen below. The sets $\{0,1 / 2\},\left\{T^{-k}(1 / 4): k=0, \ldots, 3 N_{m}\right\}$, and $\left\{T^{-k^{\prime}}(3 / 4): k^{\prime}=0, \ldots, 3 N_{m}^{\prime}\right\}$ are disjoint. Therefore, we can choose $\Delta_{m}$ and $\Delta_{m}^{\prime}$ such that the sets

$$
\begin{gathered}
\{0,1 / 2\}, T^{-k}\left(\mathcal{S}_{1 / 4, m}\right), k=0, \ldots, 3 N_{m}, \text { and } \\
T^{-k^{\prime}}\left(\mathcal{S}_{3 / 4, m}^{\prime}\right), k^{\prime}=0, \ldots, 3 N_{m}^{\prime} \text { are all disjoint. }
\end{gathered}
$$

Set

$$
f^{m}=\sum_{k=1}^{N_{m}}\left(T^{k} \bar{f}_{1 / 4, \Delta_{m}, B_{m}}-T^{k+N_{m}} \bar{f}_{1 / 4, \Delta_{m}, B_{m}}\right) .
$$

and

$$
g^{m}=\sum_{k^{\prime}=1}^{N_{m}^{\prime}}\left(T^{k^{\prime}} \bar{f}_{3 / 4, \Delta_{m}^{\prime}, B_{m}^{\prime}}-T^{k^{\prime}+N_{m}^{\prime}} \bar{f}_{3 / 4, \Delta_{m}^{\prime}, B_{m}^{\prime}}\right)
$$

We have $f^{m}=v^{m}-T v^{m}$ and $g^{m}=w^{m}-T w^{m}$ with $v^{m}=\sum_{l=1}^{2 N_{m}-1}\left(N_{m}-\mid N_{m}-\right.$ $l \mid) T^{l} \bar{f}_{1 / 4, \Delta_{m}, B_{m}}$ and $w^{m}=\sum_{l=1}^{2 N_{m}^{\prime}-1}\left(N_{m}^{\prime}-\left|N_{m}^{\prime}-l\right|\right) T^{l} \bar{f}_{3 / 4, \Delta_{m}^{\prime}, B_{m}^{\prime}}$. By (53) we have $\left\|f^{m}\right\|_{\infty}=B_{m}=1 / 2$ and $\left\|g^{m}\right\|_{\infty}=B_{m}^{\prime}=1 / 20$.

Choose $i_{m}, j_{m} \in \mathbb{N}$ such that $x_{m}=\left(i_{m}+\frac{1}{4}\right) 2^{-N_{m}}$ and $y_{m}=\left(j_{m}+\frac{3}{4}\right) 2^{-N_{m}^{\prime}}$ satisfy (51). Then

$$
\begin{gathered}
f_{N_{m}}^{m}\left(x_{m}\right)=N_{m} B_{m}, \quad\left|g_{N_{m}}^{m}(x)\right| \leq 2 N_{m} B_{m}^{\prime}=N_{m} \frac{B_{m}}{5}, \quad \forall x \in \mathbb{T}, \\
\text { and if } x \notin \bigcup_{k=1}^{3 N_{m}} T^{-k}\left(\mathcal{S}_{1 / 4, m}\right) \text { then } f_{N_{m}}^{m}(x)=0 .
\end{gathered}
$$

Similarly,

$$
g_{N_{m}^{\prime}}^{m}\left(y_{m}\right)=N_{m}^{\prime} B_{m}^{\prime}=10 N_{m} B_{m}, \quad\left|f_{N_{m}^{\prime}}^{m}(x)\right| \leq 2 N_{m} B_{m}, \quad \forall x \in \mathbb{T},
$$




$$
\text { and if } x \notin \bigcup_{k=1}^{3 N_{m}^{\prime}} T^{-k}\left(\mathcal{S}_{3 / 4, m}^{\prime}\right) \text { then } g_{N_{m}^{\prime}}^{m}(x)=0 \text {. }
$$

Let $\bar{f}^{m}=f^{m}+g^{m}$. Then $\left\|\bar{f}^{m}\right\|_{\infty}<1$ and from (54) and (55) it follows that for all $x \notin \cup_{k=1}^{3 N_{m}} T^{-k}\left(\mathcal{S}_{1 / 4, m}\right)$

$$
\bar{f}_{N_{m}}^{m}\left(x_{m}\right)-\bar{f}_{N_{m}}^{m}(x) \geq \frac{N_{m} B_{m}}{2}>m^{2}
$$

and for all $x \notin \cup_{k=1}^{3 N_{m}^{\prime}} T^{-k}\left(\mathcal{S}_{3 / 4, m}^{\prime}\right)$

$$
\bar{f}_{N_{m}^{\prime}}^{m}\left(y_{m}\right)-\bar{f}_{N_{m}^{\prime}}^{m}(x) \geq 5 N_{m} B_{m}>m^{2} .
$$

By (53) we infer (52).

Proof of Theorem 14. We will use the dense set of Lipschitz continuous functions $\left\{h^{l}\right\}_{l=1}^{\infty}$ introduced at the beginning of this subsection.

Given $l, n \in \mathbb{N}$ choose

$$
m(l, n) \geq\left(2+2 C_{l}+2 L_{l}\right) n
$$

By applying Lemma 20 with $z=z_{l}$ and $m=m(l, n)$ we choose $\bar{f}^{m(l, n)}$ which will be denoted by $f^{l, n}$. We have $\left\|f^{l, n}\right\|_{\infty}<1$ and there exist integers $N_{m(l, n)}, N_{m(l, n)}^{\prime}>$ $(m(l, n))^{2}$ and points $x_{l, n}, y_{l, n}$ satisfying $\left|x_{l, n}-z_{l}\right|<2^{-N_{m(l, n)}},\left|y_{l, n}-z_{l}\right|<2^{-N_{m(l, n)}^{\prime}}$ and such that for all $x \in \mathbb{T}$

$$
\max \left\{f_{N_{m(l, n)}}^{l, n}\left(x_{l, n}\right)-f_{N_{m(l, n)}}^{l, n}(x), f_{N_{m(l, n)}^{\prime}}^{l, n}\left(y_{l, n}\right)-f_{N_{m(l, n)}^{\prime}}^{l, n}(x)\right\}>(m(l, n))^{2} .
$$

By the Lipschitz properties of $h^{l}$ we have

$$
\left|h^{l}\left(T^{k} x_{l, n}\right)-h^{l}\left(T^{k} z_{l}\right)\right| \leq L_{l} 2^{-N_{m(l, n)}+k} \text { for } k=0, \ldots, N_{m(l, n)}
$$

and

$$
\left|h^{l}\left(T^{k} y_{l, n}\right)-h^{l}\left(T^{k} z_{l}\right)\right| \leq L_{l} 2^{-N_{m(l, n)}^{\prime}+k} \text { for } k=0, \ldots, N_{m(l, n)}^{\prime} .
$$

Therefore, by (50) for all $x \in \mathbb{T}$ we have

$$
h_{N_{m(l, n)}}^{l}(x) \leq h_{N_{m(l, n)}}^{l}\left(x_{l, n}\right)+L_{l}+C_{l}
$$

and

$$
h_{N_{m(l, n)}^{\prime}}^{l}(x) \leq h_{N_{m(l, n)}^{\prime}}^{l}\left(y_{l, n}\right)+L_{l}+C_{l} .
$$

Set now $\widetilde{f}^{l, n}=h^{l}+\frac{f^{l, n}}{n}$. By (56), (57) and (58) we have for all $x \in \mathbb{T}$

$$
\max \left\{\widetilde{f}_{N_{m(l, n)}}^{l, n}\left(x_{l, n}\right)-\widetilde{f}_{N_{m(l, n)}}^{l, n}(x), \widetilde{f}_{N_{m(l, n)}^{\prime}}^{,, n}\left(y_{l, n}\right)-\widetilde{f}_{N_{m(l, n)}^{\prime}}^{,, n}(x)\right\}>\frac{m(l, n)}{2} \geq n .
$$

Choose $\varepsilon_{l, n}>0$ such that for all $f \in B\left(\widetilde{f}^{l, n}, \varepsilon_{l, n}\right)$ we have

$$
\max \left\{f_{N_{m(l, n)}}\left(x_{l, n}\right)-f_{N_{m(l, n)}}(x), f_{N_{m(l, n)}^{\prime}}\left(y_{l, n}\right)-f_{N_{m(l, n)}^{\prime}}(x)\right\} \geq n .
$$

We define $\mathcal{G}_{n}$ and $\mathcal{G}$ as in (49) and this concludes the proof of the theorem. 
7. Complementary results and questions

Coming back to the case of an irrational rotation, for $f \in C(\mathbb{T})$, introduce the Borel set of maximizing points

$$
P_{\max }(f)=\left\{x \in \mathbb{T} \mid \sup _{y \in \mathbb{T}, n \geq 1} f_{n}(y)-f_{n}(x)<+\infty\right\} .
$$

First we have the following result:

Proposition 21. Let $f \in C_{m 0}(\mathbb{T})$ be such that $\lambda\left(P_{\max }(f)\right)>0$. Then $f=g-T g$ for some $g \in C(\mathbb{T})$.

Proof. As $f$ is bounded, $P_{\max }(f)$ is $T$-invariant. By ergodicity, a.e $x$ is then maximizing for $f$. For $M \geq 0$, let $A_{M}=\{x: S M(f, x) \leq M\}$. Since $\lambda\left(A_{M}\right) \rightarrow 1$, as $M \rightarrow+\infty$, we choose $M$ such that $\lambda\left(A_{M}\right)>1 / 2$. Fix now $y \in \mathbb{T}$. We have $\lambda\left(A_{M} \cap\left(A_{M}-y\right)\right)>0$, therefore there exists $x \in A_{M}$ such that $y+x \in A_{M}$. As a result

$$
\forall z \in \mathbb{T}, \forall n \geq 1, f_{n}(z)-f_{n}(x) \leq M \text { and } f_{n}(z)-f_{n}(y+x) \leq M
$$

Taking $z=x+y$ in the first inequality and $z=x$ in the second one, we obtain

$$
\forall n \geq 1,\left|f_{n}(x)-f_{n}(y+x)\right| \leq M .
$$

Using the cocycle property and the continuity of $f$, we arrive at

$$
\forall z \in \mathbb{T}, \forall n \geq 1,\left|f_{n}(z)-f_{n}(y+z)\right| \leq 2 M .
$$

As $M$ is independent of $y$, we obtain that $\left|f_{n}(z)-f_{n}(t)\right| \leq 2 M$, for all $n \geq 1,(z, t) \in \mathbb{T}^{2}$. Since $f_{n}\left(t_{n}\right)=0$ for some $t_{n}$, we have $\left|f_{n}(z)\right| \leq 2 M$, for all $n \geq 1, z \in \mathbb{T}$. By Gottschalk and Hedlund's Theorem (cf [13], Theorem 14.11), $f$ is a continuous coboundary.

Corollary 22. For all the $f$ in Theorems 4 and 10, $P_{\max }(f)$ has Lebesgue measure zero.

From the $T$-invariance of $P_{\max }(f)$, if $x \in P_{\max }(f)$, then its orbit $O r b(x)$ is contained in $P_{\max }(f)$. We show below that $P_{\max }(f)$ can be restricted to a single orbit.

Proposition 23. Let $\alpha \notin \mathbb{Q}$ and $T=T_{\alpha}$. Then there exists $f \in C(\mathbb{T})$ of the form $f=g-T g$, with $g \in \cap_{1 \leq p<\infty} L^{p}(\mathbb{T}) \backslash L^{\infty}(\mathbb{T})$ such that 0 is exactly maximizing for $f$ and $P_{\max }(f)=\operatorname{Orb}(0)$.

Proof. Set $B_{m}=\rho^{m}$, with $0<\rho<1$ small enough so that for all $m \geq 1$

$$
\sum_{l>m} B_{l} \leq B_{m} / 100
$$

Set next $N_{m}=1 /\left(\rho^{m} \delta^{m}\right) \in \mathbb{N}$, with $0<\delta<1$ small enough so that for all $m \geq 1$

$$
\sum_{l<m} N_{l} B_{l} \leq N_{m} B_{m} / 100
$$

We will determine the sufficiently small $\Delta_{m}$ later. 
As in the proof of Theorem 4, case $i$ ), let us define $f=\sum_{m>1} f^{m}$, where for each $m \geq 1, f^{m}$ is given by Lemma 15 via quantities $N_{m}, B_{m}$ and $\Delta_{m}$. By Lemma $15, f$ will be continuous. Fix $C>-\log (\rho \delta) / \log ((1+\sqrt{5}) / 2)$. Let now $F_{m}=\cup_{-N_{m} \leq l<N_{m}}\left[-\Delta_{m}, \Delta_{m}\right]+$ $l \alpha$, where $\Delta_{m}>0$ is such that the intervals forming $F_{m}$ are disjoint and $\Delta_{m}$ is small enough so as to ensure that $f=g-T g$ with $g \in \cap_{1 \leq p<\infty} L^{p}(\mathbb{T}) \backslash L^{\infty}(\mathbb{T}$ ) (as in the proof of Theorem $4 i$ )). We also assume that

$$
0<\Delta_{m}<\inf _{l \in[C m, C(m+1)]} \frac{1}{8}\left\|q_{l} \alpha\right\| .
$$

If $x \notin F_{m}$, via Lemma 15 , we have $f_{N_{m}}^{m}(0)=N_{m} B_{m}=\delta^{-m}, f_{N_{m}}^{m}(x) \leq 0$ and, using (59) and (60)

$$
\left|\sum_{l>m}\left(f_{N_{m}}^{l}(x)-f_{N_{m}}^{l}(0)\right)\right| \leq 2\left(\sum_{l>m} B_{l}\right) N_{m} \leq N_{m} B_{m} / 50
$$

together with

$$
\left|\sum_{l<m}\left(f_{N_{m}}^{l}(x)-f_{N_{m}}^{l}(0)\right)\right| \leq 2 \sum_{l<m} N_{l} B_{l} \leq N_{m} B_{m} / 50 .
$$

As a result, $f_{N_{m}}(x)-f_{N_{m}}(0) \leq-N_{m} B_{m} / 2=-\delta^{-m} / 2$. Consequently, if $x \notin F_{m}$ for infinitely many $m$, then $x$ is not maximizing for $f$.

In order to conclude, it therefore remains to show that $\lim \inf F_{m} \subset \mathbb{Z} \alpha \bmod 1$. Let then $x \in \liminf F_{m}$. There exists then $m_{0}$ such that for all $m \geq m_{0}, x \in F_{m}$ and thus we can find $k_{m}$ with $\left|k_{m}\right| \leq N_{m}$ such that $x \in k_{m} \alpha+\left[-\Delta_{m}, \Delta_{m}\right]$. Fix now $l \geq C m_{0}$ and choose $m \geq m_{0}$ such that $l \in[C m, C(m+1)]$. Using (61), we have

$$
\left\|q_{l} x\right\| \leq k_{m}\left\|q_{l} \alpha\right\|+q_{l} \Delta_{m} \leq N_{m}\left\|q_{l} \alpha\right\|+\frac{1}{8} q_{l}\left\|q_{l} \alpha\right\| \leq\left((\rho \delta)^{-m}+\frac{1}{8} q_{l}\right)\left\|q_{l} \alpha\right\| .
$$

For some $m_{1} \geq \max \left\{m_{0}, 8\right\}$ for any $m \geq m_{1}$ we have $(\rho \delta)^{-m} \leq((1+\sqrt{5}) / 2)^{C m} / m \leq$ $((1+\sqrt{5}) / 2)^{l} / 8 \leq q_{l} / 8$, we deduce that for all $l \geq C m_{1},\left\|q_{l} x\right\| \leq q_{l}\left\|q_{l} \alpha\right\| / 4$. Applying a result of C. Kraaikamp and P. Liardet [16], we obtain that $x \in \mathbb{Z} \alpha \bmod 1$.

We now list some questions concerning maximizing points:

- The present results should naturally extend to the context of an ergodic translation on a compact group. What can be said for distal dynamical systems? Interval exchange transformations?

- For $\left(\mathbb{T}, T_{\alpha}\right)$, with $\alpha \notin \mathbb{Q}$, it would be interesting to investigate the genericity problem for some natural non-separable subspaces of $C(T)$, such as Hölder or Lipschitz.

- Let us discuss the existence of real-analytic examples for $\left(\mathbb{T}, T_{\alpha}\right)$, with $\alpha \notin \mathbb{Q}$. For a dense set of Liouvillian angles $\alpha$ one can find a real-analytic $f$ of the form $f=\sum_{m \geq 1} f^{m}$ such that 0 is maximizing for $f$, but $f$ is not a continuous coboundary. Indeed, one may recursively build together the $\left(f^{m}\right)$ and the partial quotients $\left(a_{m}\right)$ 
of $\alpha$. As in the proof of Theorem 10,i) and using the same notation (taking $m \geq m_{0}$ and then $\varphi(m)=m$ ), for each $m$ start with some $f^{m}=H^{m}-T H^{m}$ and next take a trigonometric polynomial approximation of the peak $h^{m}$ so that $\left\|H^{m}\right\|_{\infty}$ and $S M\left(H^{m}-T H^{m}, 0\right)=\sup _{x, y \in \mathbb{T}} H^{m}(x)-H^{m}(x+y)+H^{m}(y)-H^{m}(0)$ do not vary much. Then the key estimates like (45) are uniform on all $\alpha$ such that $\left(a_{k}\right)_{k \leq m}$ are fixed and $a_{m+1}$ is large enough. Successively, one may then take for each $m$ a huge $a_{m+1}$ such that $f$ is finally real-analytic. However one has a priori no control on the degree of Liouvillianness of $\alpha$. Can one build some $f$ with the same property for any $\alpha$ verifying for example $\sup _{m}\left(\log a_{m+1}\right) / q_{m}=+\infty$ ?

- In Theorem $10 \mathrm{i}$ ), can one produce an example with an explicit upper-bound on $S M(f, 0)$ in terms of the norms $\left\|f^{(l)}\right\|_{\infty}$ ?

For lower bounds, let us make the following remark for $C^{2}(\mathbb{T})$-coboundaries.

Lemma 24. Let $f \in C^{2}(\mathbb{T})$ and $x_{0} \in \mathbb{T}$ be such that $f^{\prime}\left(x_{0}\right)=0$. Then

$$
\frac{\left\|f^{\prime}\right\|_{\infty}^{2}}{4\left\|f^{\prime \prime}\right\|_{\infty}} \leq S M\left(f-T f, x_{0}\right) .
$$

Proof. We use that $S M\left(f-T f, x_{0}\right)=\sup _{x, y \in \mathbb{T}} f(x)-f(x+y)-\left(f\left(x_{0}\right)-f\left(x_{0}+y\right)\right)$. Let $x \in \mathbb{T}$ be fixed and $|y| \leq 1 / 2$. By using Taylor's expansion

$$
f(x)-f(x+y) \geq-y f^{\prime}(x)-\left(y^{2} / 2\right)\left\|f^{\prime \prime}\right\|_{\infty},
$$

as well as

$$
-f\left(x_{0}\right)+f\left(x_{0}+y\right) \geq-\left(y^{2} / 2\right)\left\|f^{\prime \prime}\right\|_{\infty} .
$$

Taking $y=-\operatorname{sign}\left(f^{\prime}(x)\right)\left\|f^{\prime}\right\|_{\infty} /\left(2\left\|f^{\prime \prime}\right\|_{\infty}\right) \in[-1 / 2,1 / 2]$ gives the lower bound $\left|f^{\prime}(x)\right|\left\|f^{\prime}\right\|_{\infty} /\left(2\left\|f^{\prime \prime}\right\|_{\infty}\right)-\left\|f^{\prime}\right\|_{\infty}^{2} /\left(4\left\|f^{\prime \prime}\right\|_{\infty}\right)$. We maximize in $x$ to conclude.

- If $f \in C(\mathbb{T})$ admits a maximizing point $x_{0}$, can one approximate $f$ by some coboundary $f^{m}=g^{m}-T g^{m}, g^{m} \in C(\mathbb{T})$, in such a way that $S M\left(f^{m}, x_{0}\right) \leq$ $S M\left(f, x_{0}\right)$ ? Similarly, can one write $f=\sum g^{m}-T g^{m}$ with $\sum\left\|g^{m}-T g^{m}\right\|_{\infty}<\infty$ and $\sum S M\left(g^{m}-T g^{m}, x_{0}\right)<\infty$ ?

Acknowledgement. The authors thank Jean-Pierre Conze for the introduction to the question of maximizing points.

\section{REFERENCES}

[1] J. Aaronson, M. Lemańczyk, C. Mauduit and H. NAKada, Koksma's inequality and group extensions of Kronecker transformations, Algorithmics, Dynamics and Fractals, Ed. Y. Takahashi, Plenum Press, New York 1995.

[2] V. ARnold, Small denominators. I. Mapping the circle onto itself, Izv. Akad. Nauk SSSR Ser. Mat. 25 (1961), 21-86.

Prepared using etds.cls 
[3] T. Bousch, Le Poisson n'a pas d'arêtes, Annales de l'I.H.P., Prob/Stat 36 (2000), pp. 489-508.

[4] T. Bousch, Le lemme de Mané-Conze-Guivarc'h pour les systèmes amphidynamiques rectifiables, preprint.

[5] T. Bousch And O. Jenkinson, Cohomolgy Classes of Dynamically non-negative C ${ }^{k}$ Functions, Invent. Math. 148 (2002), no. 1, 207-217..

[6] J. BrÉmont, Ergodic non-abelian smooth extensions of an irrational rotation, J. of the London Math. Soc. (2010), 81: 457-476.

[7] F.E. BRowDER, On the iteration of transformations in noncompact minimal dynamical systems. Proc. Amer. Math. Soc. 9 (1958), 773-780.

[8] D.A. Carlson, A.B. Haurie And A. Leizarowitz, Infinite horizon optimal control: deterministic and stochastic systems, Springer (1991).

[9] J.-P. Conze, Recurrence, ergodicity and invariant measures for cocycles over a rotation, Contemporary Mathematics, vol. 485 (2009), 45-70.

[10] J.-P. CONZE AND Y. GuIVARC'H, Croissance des sommes ergodiques et principe variationnel, unpublished manuscript, Rennes I.

[11] A. Cohen And J.-P. Conze, Régularité des bases d'ondelettes et mesures ergodiques, Revista Mat. Iberoam., vol. 8, num. 3, (1992).

[12] J. FELDman AND C. Moore, Ergodic equivalence relations, cohomology and von Neumann algebras, I. Trans. Amer. Math. Soc. 234 (1977), no.2, 289-324.

[13] W. Gottschalk and G. Hedlund, Topological Dynamics, Amer. Math. Soc. Colloq. Publ. 36, Providence, RI, (1955).

[14] O. Jenkinson, Ergodic Optimization, Discrete and Continuous Dynamical Systems 15 (2006), pp. $197-224$.

[15] A.YA. Khinchin, Continued Fractions, The University of Chicago Press, Chicago, IL, (1935).

[16] C. KraAikamp and P. Liardet, Good approximations and continued fractions, Proc. A. M. S., vol. 112, no. 2, 1991, p. 303-309.

[17] J.C. LAGARIAS AND Y. WANG, The finiteness conjecture for the generalized spectral radius of a set of matrices, Linear Algebra Appl. 214 (1995), pp. 17-42.

[18] M. Lemańczyk, F. Parreau And D. Volný, Ergodic properties of real cocycles and pseudohomogeneous Banach spaces, Trans. of the AMS, vol. 348, no. 12, (1996), 4919-4938.

[19] M. Lin AND R. Sine, Ergodic theory and the functional equation $(I-T) x=y$, J. Operator Theory 10 (1983), no. 1, 153-166.

[20] R. MANÉ, Generic properties of problems of minimizing measures of Lagrangian systems, Nonlinearity 9 (1996), pp 273-310.

[21] A. RÉNYI, Probability Theory, North-Holland Series in Applied Mathematics and Mechanics, vol. 10, North-Holland, Amsterdam-London, 1970; German version 1962, French version 1966, new Hungarian edition 1965.

[22] K. Schmidt, Lectures on Cocycles of Ergodic Transformations Groups, Lecture Notes in Math. vol. 1, Mac Millan Co. of India (1977). 